\title{
Experiencing, Psychopathology, and the Tripartite Mind
}

\author{
Douglas Ozier ${ }^{1}$, Chris Westbury ${ }^{2}$ \\ ${ }^{1}$ Department of Education, University of Alberta, Edmonton, Canada \\ ${ }^{2}$ Department of Psychology, University of Alberta, Edmonton, Canada \\ Email: dozier@ualberta.ca,chrisw@ualberta.ca
}

Received February 7, 2013; revised March 24, 2013; accepted May 7, 2013

Copyright (C) 2013 Douglas Ozier, Chris Westbury. This is an open access article distributed under the Creative Commons Attribution License, which permits unrestricted use, distribution, and reproduction in any medium, provided the original work is properly cited.

\begin{abstract}
The philosopher Eugene Gendlin argues that a distinctive mode of reasoning, called experiencing, is necessary for working through personally salient problems such as are encountered in psychotherapy. We review supporting empirical support. It is now possible to consider Gendlin's ideas from a neurological perspective. Work directed at understanding the neurological underpinnings of consciousness and self-related processing, as well as comparative neuroanatomical work, are all consistent with and elucidated by Gendlin's experiencing construct. We argue from this data that the human mind is composed of three interacting systems that are unique to or enhanced in humans compared to other primates. Two are dedicated to "hot and cold" cognition. The most important, least well-studied third system is dedicated to mediating between these forms of cognition. We outline how interactions between these systems define different forms of psychopathology and what they suggest about the structure of the human mind.
\end{abstract}

Keywords: Emotion; Consciousness; Eugene Gendlin; Experiencing; Neuropsychology; Psychopathology; Psychotherapy; Self-Related Processing; Embodied Cognition; Phenomenology

\section{Introduction}

Based on clinical observation, psychotherapist and philosopher Eugene Gendlin put forward a view of human psychological processing that has clear implications for neuropsychological functioning, though it takes no direct inspiration from that field. In this paper we outline these implications and the evidence supporting them. Much relevant work has been done in recent years in trying to understand the neurological networks involved in generating complex, integrated, and embodied models of the self. We review empirical evidence from those and related studies to develop a neurologically grounded model of what happens to a client during a successful psychotherapeutic experience. This model has significance both for understanding the brain processes that may underlie therapeutic change and for informing clinical psychotherapeutic practice.

We begin with a very brief introduction to and overview of psychotherapy itself.

\section{What Is Psychotherapy?}

Stripped to its essentials, psychotherapy, in all its manifestations, may be characterized as the attempt to change a client's sense of experienced meaning. Although psy- chological and behavioral manifestations of symptoms vary widely, in every case in which psychotherapy is a feasible treatment option, the client wishes to alter her phenomenological experience.

While schools of psychotherapy continue to multiply, there has long been opinion $[1,2]$ and more recently much empirical evidence (reviewed below) suggesting that there may be common factors underlying all psychotherapeutic processes, regardless of their theoretical underpinnings. This topic was first broached by Rosenzweig in 1936 [3]. His claim that effective psychotherapeutic encounters are effective because they share common elements has been referred to as "the Dodo Bird Verdict" [4] since a Dodo bird claimed in Lewis Carroll's (1865) Alice in Wonderland [5] that: "Everybody has won, and all must have prizes".

Evidence in favor of the Dodo Bird verdict comes from the fact that many impartial meta-analyses of psychotherapy outcome studies have found that all common schools of psychotherapy have approximately equal effect sizes [6-8] (for discussions of the role of bias in studies that conclude otherwise, see [9-11]). This conclusion does not mean that psychotherapy is ineffective: the effect sizes are not equal because they are very low. The average psychotherapy effect sizes in meta-analysis of outcome 
studies of therapy exceed 0.85 [6,7], unquestionably large effect sizes.

Given the evidence for common factors in successful psychotherapy, a great deal of psychotherapy process/ outcome research has been devoted to trying to understand what those common factors might be. Some of the earliest of this process/outcome research was conducted in the early 1960s under the direction of Eugene Gendlin, who was trained as a phenomenological philosopher in the lineage of the French philosopher Maurice MerleauPonty. Gendlin investigated the processes through which human beings use language in order to generate fresh ideas in a creative, emergent manner; meaning making processes that allow for the resolution of complex, ill defined problems. Gendlin began studying the process of psychotherapy because that process provided him with an ideal means of investigating the phenomena of interest [12].

In their early process/outcome work, Gendlin and his colleagues collected hundreds of hours of audiotapes from completed therapies [12], rating each treatment for degree of outcome success. The researchers analyzed the tapes to try to identify in-session behaviors that predicted therapeutic success. Gendlin hypothesized that there would be specific therapist behaviors and/or certain topics of discussion that would predict therapeutic outcome. However, no such mediator variables were identified. Instead, he noticed that there was something about how the clients talked that seemed to differentiate successful from nonsuccessful therapies. Successful clients routinely spoke in a distinctive, slow, tentative, halting manner, as if they were frequently confirming the rightness of what they had just said against some kind of inchoate, background sense of what they meant to say (though they hadn't actually said it yet).

In response to these observations, Gendlin developed a theory of human cognitive function centered on the distinctive mode of reasoning he felt he had discovered, a reasoning process that he subsequently labeled experiencing. Since that time, a substantial body of empirical evidence has emerged to support the claim that experiencing does play a key role in psychotherapeutic success. Depth of client experiencing is one of the few process variables that correlates consistently with positive therapy outcome [13-15].

Most of the therapy process/outcome studies looking at the experiencing variable have used the EXP Scale $[16,17]$, "widely considered to be the gold standard of good experiential process and [...] one of the most extensively studied and validated measures of productive in session process in psychotherapy research..." ([18], p. 117). High EXP scores have " $\ldots$ been shown to be predictive of good treatment outcome across most major schools of psychotherapy, including client-centered the- rapy, CBT, psychodynamic therapy, and emotion-focused psychotherapy." ([18], p. 117). Strikingly, one study [19] found that depth of client experiencing was a better predictor of success than the strength of the therapeutic alliance, a robust and widely cited mediating variable. Depth of client experiencing therefore qualifies as one of a handful of mediating variables that have been shown to be psychotherapeutic common factors.

Gendlin [20] argues that, while depth of experiencing is crucial to psychotherapeutic change, it is not limited to psychotherapy. Experiencing is posited to be a widely prevalent, innate form of cognitive processing that is well suited to helping human beings reason through complex, ill-defined, personally salient problems. If it is true that experiencing is an innate human capacity, then it follows that it should be conducted with the brains that human beings have evolved. Further, given the high degree of mental sophistication that Gendlin attributes to it, experiencing is likely to be supported by neural components that are either unique to human beings or that are much more highly developed in our species.

In recent years various lines of research have lent this kind of neuroscientific credibility to Gendlin's description of experiencing as a distinct form of human information processing. Chief among these areas have been research into the sense of self $[21,22]$; the role of emotion in reasoning [23,24]; and the importance of interoception/background feeling states [21,22,25].

The three central goals of this article will be:

a) To offer a cogent description of experiencing as conceived of by Gendlin

b) To outline and offer selected neuroscientific support for a tripartite model of a neural subsystems that we believe to interact in order to support different levels of experiencing

c) To offer a consideration of the implications of our model for conceptualizations of psychopathology.

\subsection{Gendlin's Model of Psychotherapy}

Explaining experiencing is made challenging by the fact that the fundamental construct upon which it rests, the felt sense, is not easily described with commonly used language. A taxonomy of emotions developed by Lane \& McRae [25] is useful in introducing the concept of the felt sense. That taxonomy draws a fundamental distinction between emotions and feelings.

Emotions are defined as non-conscious, implicitly generated visceral activations and/or action tendencies that arise in response to a stimulus. Lane \& McRae distinguish two basic classes of emotions. One class consists of discrete foundational emotional states such as anger or sadness. The other class consists of "background emotions [that are] bodily states... generated by internal regulators as well as external stimuli and [that] provide 
information about one's current state of well being" [25] (p. 100).

Feelings are defined as cognitions that arise through the explicit awareness of emotions. Lane subdivides feelings into different classes: focal, reflective, and background. Focal feelings arise through attending to foundational emotions such as fear or anger. Reflective feelings involve the conscious reflection upon focal feelings and therefore involve a significant meta-cognitive component. Background feelings arise through attending to background emotional states. Because background emotions are more diffuse then focal emotions, background feelings typically arise on the periphery of awareness as relatively diffuse conscious experiences such as "feeling lousy" [25] (p. 103). However, crucially for the model we will develop, Lane and McRae postulate that when background emotional states are given adequate attention it is possible for them to give rise to consciously accessible background feelings.

Gendlin holds that the experiencing is based on the functioning of the felt sense system, a non-consciously controlled yet highly sophisticated form of human information processing. Gendlin claims that the felt sense system underlies that aspect of the self that feels most strongly “... like 'really me"” [26] (p. 81). A specific felt sense is a "bodily sense of some situation, problem, or aspect of one's life..." [27] (p. 20). In Lane's terms, a specific felt sense is the background feeling that arises through consciously attending to the background emotion that is associated with a particular life situation. Felt senses are "implicit higher level meanings [... involving...] the sense of something that includes thoughts, feelings, perceptions, internal actions, and context." [28] (p. 165). A felt sense contains implicit meanings before they have been separated from directly lived, holistic experience and before they have been symbolized in any way. Once a felt sense has become the focus of awareness, specific focal emotions, memories, images, or ideas that are associated with particular aspects of the overall situation in question can emerge out of the felt sense. These specific phenomena can then become figural against the ground of the felt sense. However, the discrete and tangible phenomena evoked by attending to the felt sense are not themselves the felt sense.

There are two related differences between a felt sense and a focal feeling such as sadness or anger. First, as a form of background feeling, a felt sense manifests less distinctly and less intensely than a focal feeling. Second, the meaning of a focal feeling is explicit while the meaning of a felt sense is implicit. A generic symbol (such as the word "sad" or "angry") is able to describe a given focal feeling each time we feel it. A felt sense, in contrast, is difficult to symbolize. Each felt sense contains a rich, complex mosaic of implicit meanings. Successful expe- riencing, in Gendlin's sense, is the act of using language to symbolize the next step toward healing or problem resolution that will frequently be implicitly contained within a felt sense.

\subsection{Three Levels of Experiencing}

Different levels of experiencing are recognizable by their different characteristics.

\subsubsection{Low Level Experiencing}

At low levels of experiencing ( $1-3$ on the 7 point EXP Scale [16]), the client typically speaks largely from her head in a conceptual manner marked by the dominant use of an Externalizing Voice [29]. Externalizing Voice is marked by an externalizing vocal quality, with the speaker speaking at a regular, fast, even pace, indicating "... that energy is being invested in recounting rather then exploring" [30] (p. 282). During low experiencing the client quickly and effortlessly projects his sense of autobiographical self into simulated realities that are largely decoupled from the physical reality in which the client and therapist find themselves [31]. The client stands outside himself and tries to deduce what he "must" want, how he "must" be feeling about his situation, and therefore what step he "should" take next to resolve his presenting issue.

Low level experiencing is a valuable mode of processing for human beings. Its primary advantages are the rapidity and ease with which mental projections can be conducted. These qualities make it very well suited for solving problems in domains of relative certainty by rapidly generating possible future events and then thinking them through (see related discussion in [23]). However, it is notably ill suited to resolving the kinds of complex, highly embedded, value laden life challenges that usually bring people into psychotherapy. Low level experiencing is correlated with unproductive psychotherapeutic process [12]. Externalizing Voice, which is strongly correlated with low level experiencing, is also predictive of therapeutic failure [32].

\subsubsection{Medium Level Experiencing}

During medium level experiencing the client's verbal patterns typically have an irregular, bimodal quality in which the client alternates largely between the previously described Externalizing Voice and a Focused Voice [29]. Focused Voice has a tentative, inwardly searching quality, indicating a "turning inward of attentional energy toward tracking inner experience" [30] (p. 282). It tends to be slow and halting, with frequent starts and stops as possible next steps are inwardly checked for "rightness" against the implicit sense of what needs to be said next [29]. During medium level experiencing the client will frequently speak in Externalizing Voice until an emerg- 
ing feeling of wrongness implicitly tells him that he is beginning to lose his sense of "maximal grip" [33] on the topic at hand, or that he is beginning to veer off course from what he is trying to say. This veering off course will typically initiate transition to a period of Focused Voice, which has been found to be associated with productive therapeutic process [32]. Typically, when an increasing feeling of rightness eventually tells the client that he has found the thread again, this will transition back to another phase of Externalizing Voice.

As these characterizations of the process imply, at medium levels of experiencing, it is almost as if an unfelt felt sense is present. It is unfelt because the implicit whole is not accessible to awareness. It is felt because the results of unconsciously trying to speak from that implicit whole are consciously accessible, manifest in continuous scale somatic feelings of rightness or wrongness. In this mode of reasoning the rightness of the next step is, usually unconsciously, determined to a significant extent by the degree of felt rightness that emerges when each possible next step is being considered. Medium level experiencing, combined with occasional periods of high level experiencing, is typically the most productive mode of information processing during psychotherapy.

\subsubsection{High Level Experiencing}

At high levels of Experiencing (EXP Scale levels 5 to 7) the client senses her situation as a lived whole (as contained within the associated felt sense) and then experientially reasons, step by step, through her issue from inside that felt sense. In contrast to mid-level experiencing, the felt sense is now symbolized in conscious awareness and can be used in a deliberate, intentional manner as the client moves back and forth between its implicit meanings and the explicit meanings contained within proffered symbols [27,33]. The client's verbal patterns tend to be extremely slow and irregular with frequent periods of very long silence. When it does occur, speech is typically largely in the previously described Focused Voice [29]. As with medium level experiencing, continuous scale feelings of rightness and wrongness continue to guide the process of meaning making and problem resolution. However, unlike with medium level experienceing, the correctness of possible steps can now also be overtly checked in a binary yes/no fashion. This step is achieved in psychotherapy by making a checking statement and then waiting for the felt sense system to respond by either producing, or failing to produce, a distinctive form of sensation called a felt give. Gendlin describes the felt give as a subtle, pleasant, stirring sensation that is usually experienced in the stomach, chest or throat [27]. When a felt give arises in response to a checking statement, this is taken is an indication that the felt sense system has assessed the proffered symbol or potential next step to be a right match or a good next step.

According to Gendlin, during the iterative movement between a felt sense and symbolizing possible next steps, the felt sense's responses must be given priority. No matter how correct an idea, theory, or potential next step may appear conceptually, it should only be considered to be pointing in a useful therapeutic direction if it evokes a felt give when a checking statement is used to confirm its potential rightness.

High level experiencing is not easy for many people to engage in [27]. It often requires significant guidance. This is one disadvantage of higher-level experiencing in many problem-solving contexts. The main advantage of high experiencing is that it can help to turn implicit meanings into explicit meanings so they may to be worked with more fruitfully.

\section{From Clinical Psychology to Neuropsychology}

A client comes to psychotherapy in order to resolve issues that are usually not only complex and ill defined, but that are also highly salient to his sense of self. Regardless of the level of experiencing involved, psychotherapy can therefore be understood as a guided exploration of the client's sense of self. For this reason, in order to be credible, our neural model of self-referential processing during psychotherapy must be consistent with current neuroscientific understandings of how self-referential processing occurs more generally. In this section, we will briefly review studies on self-referential processing. However, the indivisible relationship between consciousness and the sense of self means that addressing self referencing from a neural perspective first requires access to a neurally grounded model of human consciousness. We therefore start by offering a synopsis of Damasio's (1999) model of consciousness [21] so that it can then serve as a foundation for the ideas that follow.

\subsection{Damasio's Model of Consciousness}

Damasio argues that the process of constructing the self starts with the generation of two separate neural maps. The first is a map of the stimuli with which a person is currently interacting. The second map represents the changing state of that person's own body as she interacts with those stimuli. This latter map of the changing state of the body, which forms the basis for what Damasio calls the proto-self, is putatively neurally encoded by a number of structures devoted to mapping and regulating the internal state of the body, including brain stem nuclei, the hypothalamus, and the insula.

According to Damasio, there is no sense of consciousness attached to the functioning of the proto-self because it is a process of unfolding without any sense that the un- 
folding belongs to anyone in particular. Consciousness arises when both first order maps get remapped into a single second order map, which thereby encodes a complete record of the proto-self changing itself as it interacts with the world. As this second order map arises it produces an ephemeral, background feeling that the changes occurring to the proxy, proto-self are happening to a self. It is as if the core self watches the changes in that are occurring to the proto-self while constantly regenerating a fresh background feeling that "this is me I am watching". This background sense of a watching self is the core self. The core self generates core consciousness, which is transient and is constantly being regenerated.

Damasio suggests that the second order map that is essential to core consciousness is likely generated by the coordinated activity of a limited number of brain centers. In a recent version of his model [34], Damasio stresses the importance of the anterior cingulate cortex (ACC) and the insula in supporting generation off the core self, arguing that the insula may be "involved more significantly than any other structure" (p. 105) in the subjective feeling processes that underpin self-awareness.

In Damasio's model of consciousness, over time we generate rich networks of self-defining memories. These networks allow for the emergence of extended consciousness and the related autobiographical sense of self. This sense of self becomes richly elaborated and extended through time. However, the autobiographical self always relies on the feeling of the core self to become activated in a meaningful way. It is the continuous, background activity of the core self that allows your self-defining autobiographical memories to feel like your memories. Without a constantly emerging background "feeling of what is happening" [21] there can be no extended consciousness.

\subsection{The Neurology of Self-Related Processing}

Recent research extends Damasio's model of the neural representation of the self by providing evidence that our self has a complex neural representation composed of several interacting processes.

Northoff, Heinzel, De Greck, Bermphol, Dobrowolny, \& Panskeep, (2006) performed a meta-analysis of 27 PET and fMRI studies that involved self-related tasks [35]. They coded the peak activations within each study and performed a hierarchical cluster analysis upon the 324 resultant clusters. This analysis revealed the presence of 3 reliable clusters (shown in Figure 1) that emerged regardless of the sensory mode of stimuli presentation. Region 1 is centered in the posterior parietal cortex. This region is implicated in the access of episodic, autobiographical memories [31]. Region 2 falls within the dorsomedial prefrontal cortex (dmPFC). Region 3 falls within the orbital medial prefrontal cortex (omPFC). The Region 3 omPFC cluster is implicated in non-symbolic self-re-

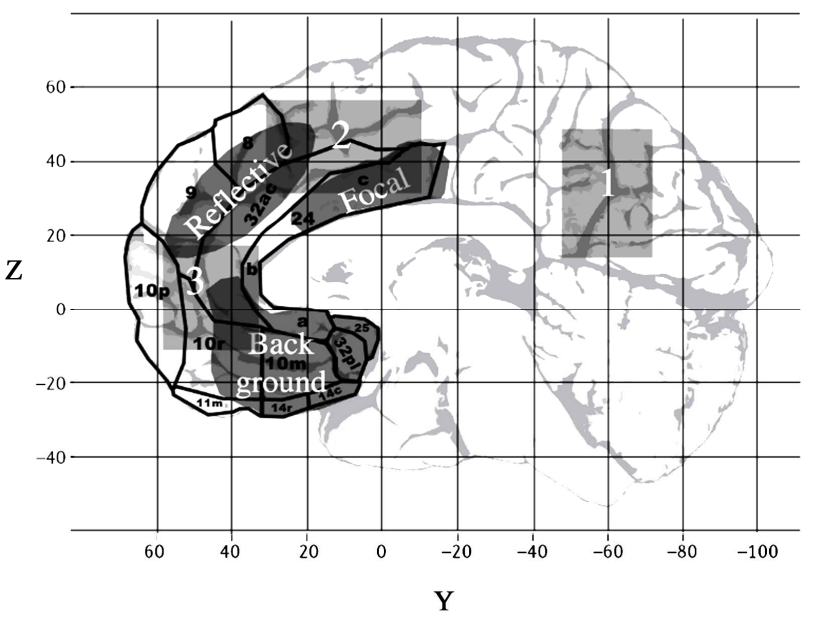

Figure 1. Summary of regions implicated in self-related tasks. The three rectangular regions are re-drawn from Northoff et al.'s (2006) meta-analysis of 27 imaging studies of selfreferential processing [35], which found three modality-insensitive activation clusters. The extent in either dimension represents the standard deviation of the cluster. Region 1 is involved in autobiographical memory. Region 2 is involved in explicit cognizing about self-related stimuli. Region 3 is implicated in non-symbolic, self-related affective processing. The three regions labeled with names are re-drawn from Lane \& MacCrae's (2003) summary of the neural substrates of conscious emotional experience [25], and are associated with the three types of emotion they identify. The area labeled "reflective" is associated with conscious reflection on experienced feelings. The area labeled "focal" is implicated in direct conscious awareness of basic emotional states such as fear or anger. The area labeled 'background' is implicated in generating background states that are not noticed unless they are attended to.

lated affective processing while the Region 2 dmPFC cluster is implicated in re-representation of this information in a way that is symbolically accessible [35].

Recent work suggests that refinement is necessary to the conclusion that there is single cluster in the omPFC that supports the "non-symbolic, affective" aspects of selfreferential processing. A study that used MRI to investigate the neural correlates of self-referential processing produced evidence that there are three neural subsystems that operate together in order to support the processes of self-reflection and self-projection [36]. These sub-systems appear to be associated with three dissociable clusters of activation within the omPFC (see Figure 2). One of these subsystems had activation centered in BA $9(0,52,26)$. This subsystem (like the more dorsal cluster in BA 8 identified in [35]) is involved in the meta-cognitive processes of reflecting upon one's own state of mind [36]. A second subsystem had omPFC activation centered in ventral BA $10(0,26,-18)$. This subsystem plays a key role in mental scene construction, a process that is vital to allowing the autobiographical self to mentally project through space and time. A third subsystem, also with 
omPFC activation, lies between the activation of the other two subsystems, in dorsal BA 10 r $(-6,52,-2)$. Activation in this region was found to be strongly associated with task variables related to "personal significance, introspection about one's own mental state, and evoked emotion" [36] (p. 558). The authors suggest that this middle subsystem is fundamentally devoted to "evaluating aspects of personal significance" [36] (p. 559) and that, of the three subsystems, it constitutes the core subsystem for self related processing.

The conclusion that self related processing is supported by three distinct PFC regions is consistent with the results of a recent resting state connectivity study de- signed to identify large, distinct functional networks within the human brain [37] (see Figure 2). That study used simultaneous EEG-fMRI in order to help overcome the difficulties that fMRI has had with distinguishing resting state networks that lie close to each other. The authors identified components of three distinct resting state brain networks within the medial PFC, arguing that the three distinct networks they identified have traditionally, mistakenly, been subsumed within a single, large, frontal component of the "Default Mode Network". The first of these networks (see gray area in Figure 2(A)) is anterior, involving clusters in the dmPFC (BA 9, centered at 9, 69, 15 ), and the OFC (BA 10p, at $0,63,-6$ ) and ventral BA

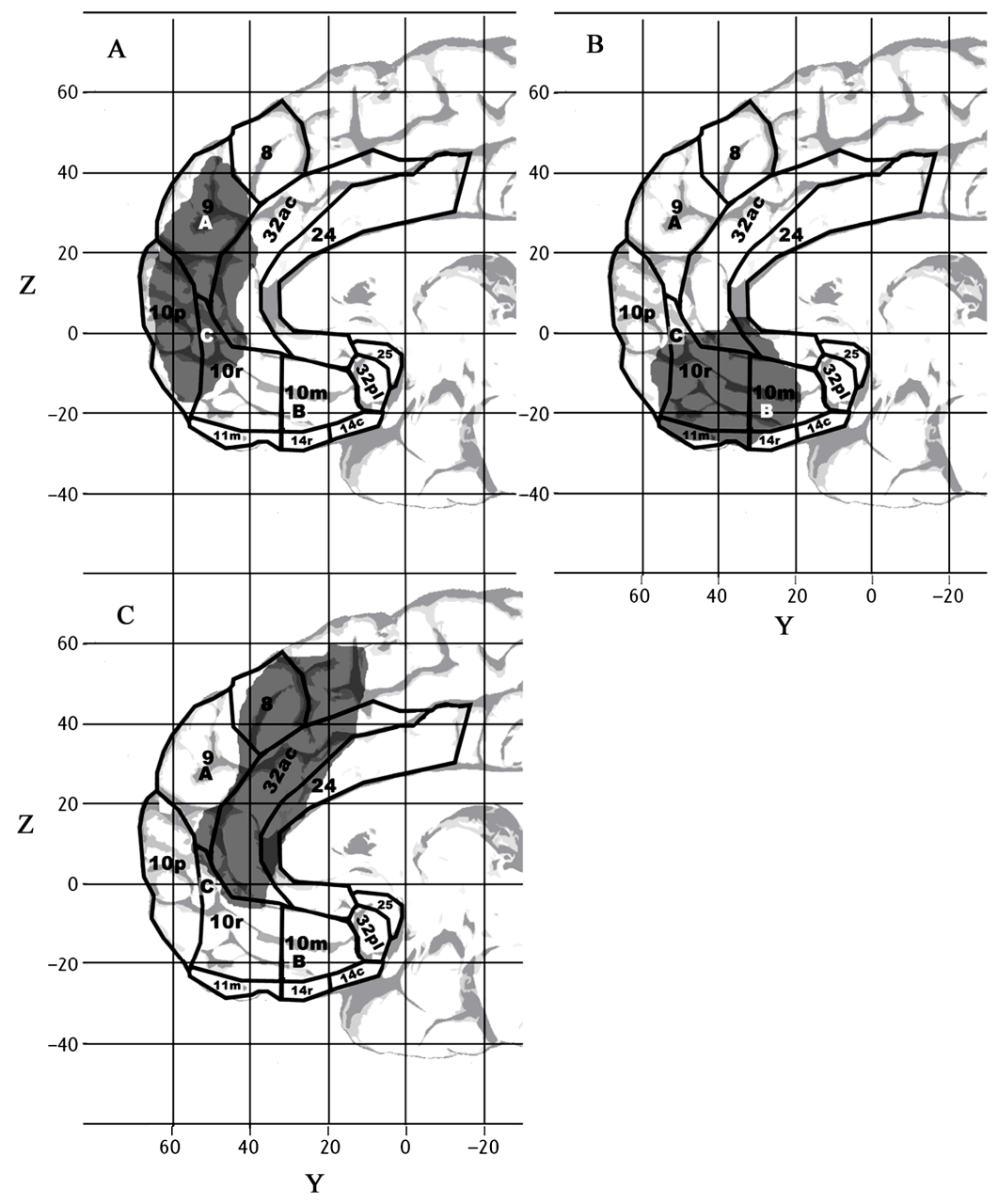

Figure 2. Frontal regions associated by resting state connectivity and with self-related processing. Grey Areas: Frontal components of the three distinct medial subsystems identified by resting state connectivity [37]. Labels A, B, \& C indicate activation centers for medial frontal activation systems associated with self-reflection and self-projection, as identified in [36]. The grey region in Figure 2A (the dorsal medial prefrontal cortex subsystem) shows functional connectivity with the temporoparietal cortex, lateral temporal cortex, and the temporal pole and is associated with meta-cognitive processes of reflecting on one's own state of mind. The grey region in 2B (the medial temporal lobe system) shows functional connectivity with the posterior inferior parietal lobe, retrosplenial cortex, and hippocampal and parahippocampal regions, and is associated with mental scene construction. The grey region in 2C, considered part of the core subsystem for self-related processing, is a common "hub" for the other two subsystems, comprised of the medial prefrontal region shown and posterior cingulate cortex, associated with introspection and evaluating aspects of personal significance. Note that all three subsystems overlap with the common hub for self-related processing [36], marked with a C. See also Figure 1. 
10 , at $9,63,-18$. The second putative resting state network identified by Liao et al. is centered in the mOFC (BAs 11 and ventral BA 10: example peak at 9, 54, -21) and overlapped the mOFC-centered subsystem identified in [36] (see gray region in Figure 2(B)). Finally, a third network lies between these two networks, centered in dorsal BA 10r and in neighboring BA 32 ac (gray region in Figure 2(C)). As shown on Figure 2, all three networks overlap with the core self-processing sub-system (labeled C in Figure 2) identified in [36].

\subsection{The Neurology of Self-Related Reasoning}

In this section we build on the ideas presented thus far in order to offer a systems level model of human, self-referential reasoning, summarized diagrammatically in Figure 3. We conceive of this model as involving interactions between three neural systems: a ventral "hot cognitive system", a dorsal "cold cognitive system", and a middle mediation system that supports middle to high level experiencing. Ultimately, we will outline the systems level interactions between these three large scale systems and how we believe these neural changes impact depth of experiencing. In advance of this we will describe the key components of each of the three posited systems independently.

We conceptualize each subsystem as involving a specific set of contextualizing neural processes (some of which also contextualize each other), where "contextualized" means that the content and output of the process is modulated (its valence and/or meaning modified) by input from another source. Each subsystem has an evolutionarily nested form, with phylogenetically older components interacting with newer analogues that perform similar functions in more complex and integrative ways. In the case of each system we will discuss these processes in phylogenetic order, first addressing the evolutionarily older components, and then their more recent analogues.

\subsubsection{Older "Hot" Cognition System}

The lowest level of our hot cognition system is formed by the basolateral complex of the amygdala (BLA, made up of the lateral, basal, and accessory basal nuclei). The amygdala is a complex, heterogeneous, and phylogenetically ancient system. For the purposes of our model (following [38]), we consider a simplified model of the amygdala consisting of the BLA and of the central nucleus (CNA).

The CNA has extensive connections to the brainstem, hypothalamus, basal forebrain, and the ventral insula, as discussed below. It plays crucial roles in determining general affective responses to both appetitive and aversive stimuli and in initiating species-specific behaviors in response to those stimuli $[39,40]$. The BLA has strong cortical connections by which it receives extensive multi- modal sensory input, and plays a crucial role in associative learning by encoding memories related to sensory stimuli [41]. In turn, this allows the BLA to modulate the “... memory encoding and sensory processing in other regions... [by broadcasting its memory-informed assessment of] the threat value of a stimulus" [41] (p. 1362). Chief among these modulated regions is the CNA itself, to which the BLA sends output. In our terms, the BLA contextualizes the output of the CNA.

\subsubsection{Newer "Hot" Cognition Subsystem}

As illustrated in Figure 3, the BLA is itself contextualized by the medial orbital PFC, to which it has strong bidirectional connections [42]. Acting in concert with adjacent vmPFC, medial orbital PFC (BA 11) may be important for using input from the amygdala to represent the pleasant or unpleasant affective value of a stimulus $[42,43]$ in a flexible format that is sensitive to momentary changes in social and motivational context $[39,44]$. This allows for a richer encoding of the affective properties of stimuli than the amygdala alone would be capable of computing $[45,46]$. We believe that it is useful to conceptualize of medial BA11 as an evolutionarily new analogue of the amygdala's BLA, since it integrates emotional memory and guides appropriate behavior to encountered stimuli.

Rolls has argued that orbital/ventro-medial PFC complex is the "computer of reward magnitude and expected reward value..." [47] (p. 114) tracking the ongoing, homeostatically contextualized, dynamic reward value of encountered stimuli. One piece of evidence cited in support of this claim is that neurons in this region fire faster in response to food when a person is hungry then when he is not. In the orbital PFC, reward valuations are ingrated with other relevant information in order to generate representations of "expected value", defined as probality times reward value [47]. These expected value reesentations are then fed more dorsally, this time into the vmPFC. There they are processed into representations of subjective utility [47]. Subjective utility integrates the expected reward value of a stimulus with broader contextual information such as the person's degree of risk aversion and other related personality and/or internally mediated factors. Reward value, expected value, and subctive utility judgments are thus neurally coded in a connuous fashion, based in part on the finding that there is a linear correlation between assessed value and the firing rate of the coding neurons [47].

Neuroimaging [48] and neuropsychological evidence [49] suggests that a medial OFC/vmPFC subsystem gerates somatic markers in order to communicate its prected value of potential rewards (see Figure 4). Somatic markers are a form of gut feeling through which vmPFC subsystem (in particular) is able to, often implicitly, guide both behavior and conscious cognitive processing [23]. 


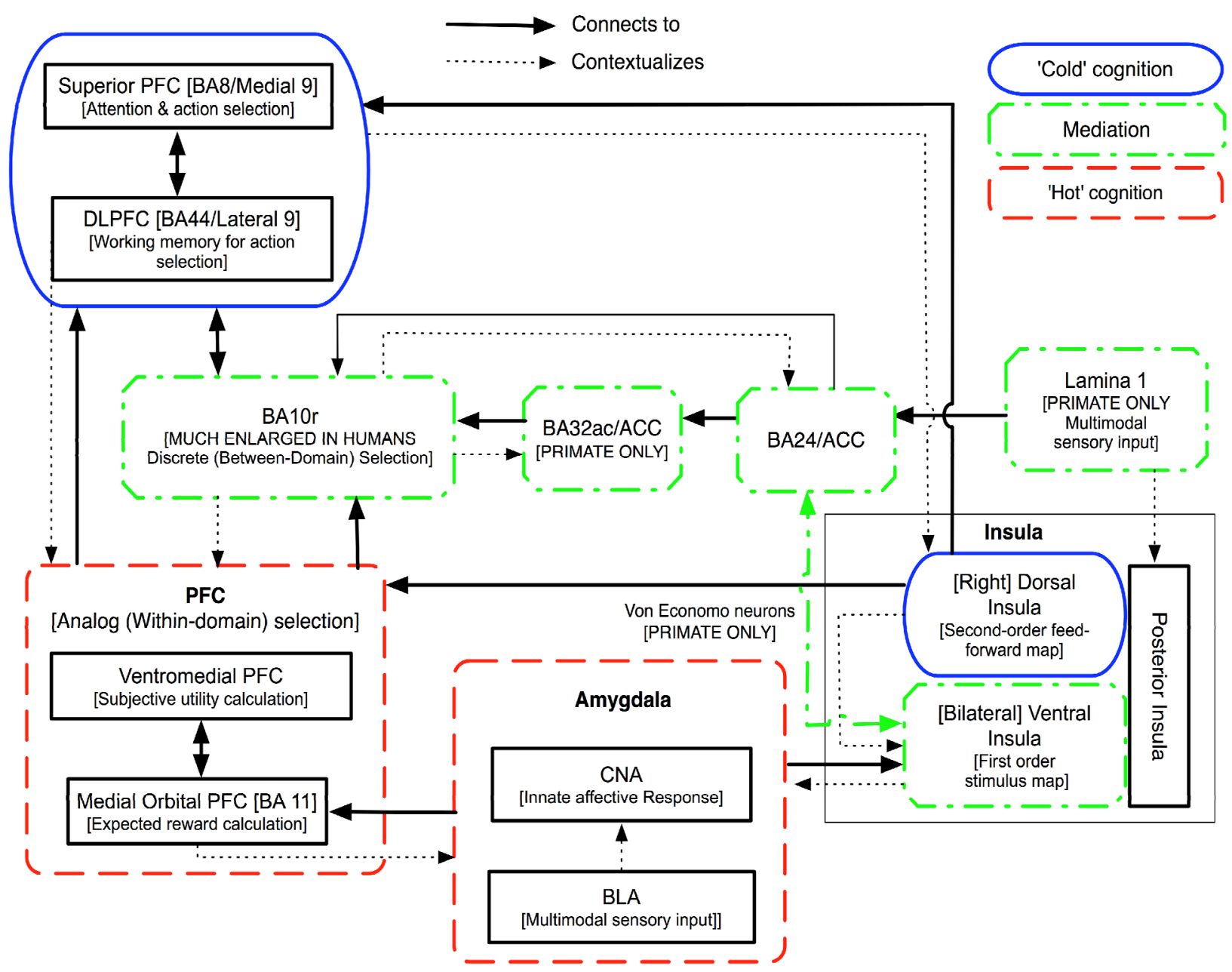

Figure 3. Outline diagram of the tripartite model of the human mind. The model outlines the neurological underpinnings of separate systems for cold and hot cognition, as well as a third system for mediating between the two.

\subsubsection{Older "Old"Cognition Subsystem}

Craig [50,51] has produced neuroanatomical and experimental evidence of the existence of a previously unrecognized afferent neural system called Lamina I that has crucial implications for our model. Lamina I only exists within human beings and other primates. It collects affrent information from all the tissues of the body and systematically integrates and represents this information. Lamina I terminates in three cortical locations [51]: somatosesnory cortex (BA 3a), dorsal ACC (BA 24c) [52], and the posterior insula [22,51]. Within the insula, Lamina I continues to feed forward until termination in the anterior insula.

The insula has been assigned a diverse range of functions. Among these have been roles in learning and memory, perception of temporal sequence, pain perception, language, autonomic arousal, and integration of emotional and motivational factors [53]. As shown in Figure 3, the insula will be presented in a simplified manner as being composed of three functional zones: the dorsal AI, the ventral AI, and the posterior insula. We will focus almost exclusively on the anterior insula here.

It is important to distinguish between ventral and dorsal AI, since these two regions have markedly different resting state connections with the PFC [54; see Figure 5]. The ventral AI (with a right ventral AI seed at 30, 23,-11) shows very limited resting stated connectivity with the lateral PFC. It does show strong resting state connectivity with the medial PFC, including with the inferior ACC (BAs 24 and 32ac) and medial PFC (BA 10). The dorsal AI (with a right seed identified at $34,29,4$ ) has strong connections with lateral PFC, including an epicenter of connectivity in the dIPFC. The dorsal AI shows strong resting state connectivity with one just medial PFC region $(0,36,46)$. This falls in a region that was identified (in [55]) as being the only medial PFC centered node within the Executive Network, a network of brain regions that is centered in the dIPFC (with bilateral epicenters in both BAs 46 and lateral BA 9) and implicated in attentional control and conscious response selection. That 


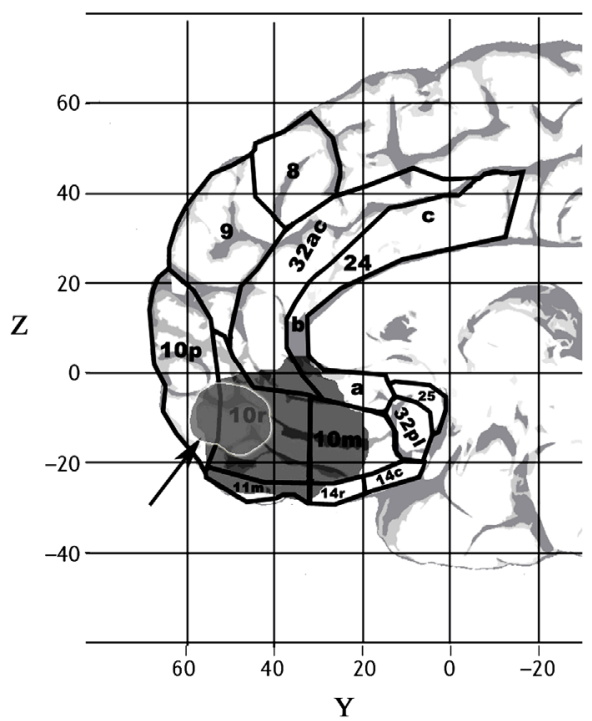

Figure 4. Region associated with reward valuation. Arrow points to medial BOLD activation in the region that was positively correlated with successful learning during completion of the Iowa Gambling task [48]. Note the overlap between this putative "somatic marking region" and the center of the ventral medial subsystem identified by resting state connectivity [37] (see Figure 2(B)).

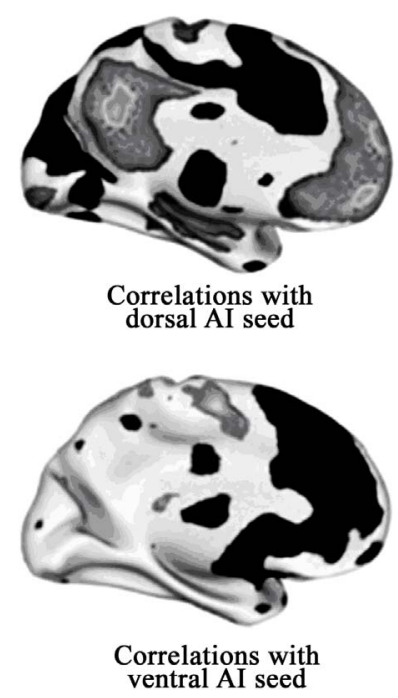

Figure 5. Resting state connectivity associated with dorsal and ventral AI, on inflated medial brain surfaces. The dorsal (top) and ventral (bottom) AI show markedly different patterns of resting state connectivity with left medial cortex (Figure adapted from [54], p. 676). Black regions are positive correlations with seed regions in the dorsal versus ventral AI; light grey regions are negative correlations with each seed.

Executive Network has strong parallels to what we are calling the cold cognition system.

A recent meta-analysis [56] provides further evidence of a functional distinction between the ventral and dorsal components of the AI. This study looked at the results of over 1700 neuroimaging studies to investigate the involvement of different insular regions within four broad functional domains. The ventral AI was implicated only in processing tasks that required social or emotional valuation. The dorsal AI was implicated in processing tasks in the same social-emotional domain but also to be strongly and broadly activated by tasks in the cognitive domain (e.g., language, attention, memory). These findings support our key contention that, along with the dIPFC and dmPFC, the dorsal AI is a part of the brain's cold cognition system. However, unlike the dIPFC and/or dmPFC, the right dorsal AI serves as the key interchange between this system and the brain's ventral AI centered mediation system, as will be discussed below.

Craig [51] argues that, as a key terminus for the Lamina I information, a consciously accessible "meta-representation" (p. 239) of the current state of the entire body is generated within the right dorsal anterior insula. According to Craig, this representation provides a person with a background sense of self as an integrated, embodied entity and is "the material me" (p. 241). This right dorsal anterior insula meta-representation therefore constitutes a second order map in Damasio's [21] terms, providing a contextual, homeostatic ground against which ongoing first order maps (representations of the protoself responding to salient stimuli) can become figural. We contend that first order, proto-self related maps are also represented in the insula, but bilaterally in the ventral AI.

The ventral AI directly receives salience assessments about encountered stimuli from the CNA [55] (see Figure 3). Via Lamina I, fast amygdala-driven assessments of the salience of an encountered stimulus can be contextualized: i.e. given greater or lesser weight. During this process, first order neural maps that include information from Lamina I about the organism's overall homeostatic state contextualize the CNA's salience assessments. These maps (supported by ventral AI) can then be immediately re-contextualized by second order maps (supported by right dorsal AI) based on Lamina I information (as will be discussed below). The ventral/dorsal AI figure-ground relationship makes it possible for homeostatic meaning to be quickly and cogently assigned to whatever internal or external stimulus is encoded in the first order body map in question.

As a concrete illustration of this process, Craig [52] asks us to compare the experience of drinking a cool glass of water on a warm, summer day with this same experience on a rainy day when we are wet and cold. This example makes clear that it is not possible to ascribe homeostatic meaning to a stimulus in itself. A stimulus must always be considered against the homeostatic context of the organism's current state. During daily life this necessity is strongly obscured by the human tendency to 
project the meaning of things onto the things themselves [52]. It is only through the crossing of the part (contextualized encoding of the body's action response to a specific stimuli) against the whole (enhanced awareness of the body's current, overall homeostatic state) that both homeostatic meaning and self-awareness of that meaning become possible [21,51].

The existence of Lamina I only in primates is suggestive evidence that primates may have evolved a unique ability to refine the CNA's preliminary salience assessments. The described process may allow primates to quickly get both a "first opinion" (supported by ventral AI) and a "second opinion" (supported by right dorsal AI) about the CNA's original interpretations. A demonstration of this capability comes from a study in which married women were threatened with electric shock under two conditions: while alone; and while holding their husband's hands [57]. Unpleasantness was significantly lower during the hand holding condition, and this was most strongly associated with lessened activity in the right anterior insula (centered at 37,16,3). Lamina I based information was putatively used to "tune" the meaning of the threat related stimuli by factoring in a socially supportive background context (see also [58] for evidence implicating the same region in the right AI, centered at 42,16,1, in the pain of social rejection). Lamina I re-contextualization allows the meaning, or threat value, of pain stimuli to be "tuned" by factoring in a background context that includes the presence of social support.

\subsubsection{Newer “Cold” Cognition Subsystem}

Findings cited in the section above have led us to designate the dIPFC (BAs 9/46) and more dorsal, medial PFC regions (BAs 8/9) as components of our evolutionarily new cold cognition system. We will focus in detail only on the dIPFC here.

The dIPFC has been implicated by human imaging and animal lesion studies in a wide range of higher cognitive functions, including evaluation of expected reward; response and goal selection, initiation, and inhibition; word and random response generation; attentional shifting; sensitivity to the demands of a changing context; and selective retrieval from a set of items held in short-term memory. Several commentators have tried to generalize across these tasks, arguing that the prefrontal cortex in general is critical "when we need to use the 'rules of the game,' internal representations of goals and the means to achieve them" ([59], p. 168); that dlPFC in particular is active when "several pieces of information in working memory need to be monitored and manipulated on the basis of the requirements of the task or the subject's current plans" ([60], p. 61); that the region's role may be characterized as "manipulation of the representation in context of the instructions and mapping the response onto an output system" ([61], p. 2195); that the dlPFC plays "a role in guiding behavior that does not ensue immediately, but is to be enacted seconds after the acquisition of a sensory instruction" ([62], p. 415); or that all tasks modulated by dIPFC "have to do with using information about something you've just done or seen against itself, so to speak, to inhibit the tendency to follow up that correlation and instead shift attention and direct action to alternative associations." ([63], p. 263). These descriptions all capture the fact that the role played by the dIPFC involves modulating the nature of imminent behavior in response to changing task demands. An elegant phrase for the role has been proposed by Frith [64]: "sculpting the response space". Although Frith's pithy description over-emphasizes the dlPFC's role as an autonomous entity while under-emphasizing the neurological context upon which it is dependent, it does capture the idea that cold cognition is in large part devoted to the slow selection of action, especially under the guidance of memory.

As we have mentioned earlier, a key element of our model is our contention is that the right dorsal AI-dlPFC relationship is bi-directional. In other words, it is not only that the right dorsal AI provides the dlPFC with the homeostatic background against which its cognitive operations can become figural, though this is required if these operations are to occur within conscious awareness. The dlPFC also feeds back to the right dorsal AI, thereby allowing the evolutionarily new cold cognition system to directly influence the contents of the brain's crucial second order body map (see Figure 3). In other words, we believe that the right dorsal AI continually and dynamically integrates real-time Lamina I information with top-down, memory-based predictions of what that the person's overall, homeostatic context will be like in the very near future. We posit that these top-down alterations are generated unconsciously and are experienced as being completely real by the person generating them [21, 65]. In short, the right dorsal AI meta-representation is a feed-forward model of the embodied sense of self [66].

Though the right dorsal AI is always integrating Lamina I real time information with top down predictions, the relative balance between these two forms of input is variable. As will be discussed at length in the final section of this paper, this balance is a key factor in determining depth of experiencing.

The top down mechanism just described strongly echoes the "as-if-body loop" construct posited by Damasio [21, 23]. Damasio claims that higher order brain centers can distort the contents of the neural maps that underlie core consciousness. These top down simulations are very fast because they are enacted entirely within the brain and do not rely on the much slower process of monitoring actual changes going on within the body proper, such as the 
ones that are reflected in Lamina I output. This "as-ifbody loop" element has two important implications. First, the integration of both Lamina I information and top down predictions greatly increases the sophistication and speed with which the right dorsal AI can tune the ventral AI's salience assessments. Second, top down input into right dorsal AI can at times be much stronger then Lamina I input. The resulting cognitive mode would allow for the fastest possible generation of the "embodied" backgrounds considered necessary for consciousness [23], thereby allowing the cold cognition system to imaginatively move through space and time with tremendous speed. This concept is essential to our conceptualizations of both low level experiencing and psychopathology and will be discussed in the final sections of this paper.

\subsubsection{Older Mediation System}

The third system in our model (Figure 3) is a mediation system that helps integrate the hot and cold cognition systems considered above. As with the hot and cold systems, we identify two sub-systems, one evolutionarily newer then the other.

In addition to identifying an "Executive System", Seeley et al. [55] also identified a large scale "Salience Network" within the brain. Their "Salience Network" is devoted to determining which stimuli are the "most homeostatically relevant" from among the endless range of "internal and extra-personal" stimuli that bombard the nervous system ([55], p. 2354). The center of the Salience Network falls in the bilateral ventral AI $(42,10$, -12 and $-40,18,-12)$. It also has key nodes in the CNA $(20,4,-20)$, anterior ACC (throughout BA 24 A and B), and the medial PFC (BA 10r; -24, 56, 10) [55]. These maxima clearly illustrate that what we have termed the "mediation network" closely follows Seeley et al.'s "Salience Network" not only in terms of function, but also in terms of brain region membership (see Figure 3).

The lowest level of the old mediation system is the amygdala's CNA. As described earlier, the CNA plays a crucial role in determining general affective responses to both appetitive and aversive stimuli [40] and in initiating species-specific behaviors in response to those stimuli [48]. In other words, the CNA helps organisms recognize what matters on a basic level and also helps to generate the embodied action tendencies needed to act effectively in response. The CNA sends its assessments to the ventral AI for further refinement as needed [55].

Recall that Lamina I terminates in the posterior insula. As a result the ventral and dorsal AI are both ideally positioned to receive input from Lamina I. The ventral AI is also strongly connected to diverse limbic and paralimbic structures including the ACC [67]. This allows ventral AI to contextualize the CNA's salience assessments and to initiate homeostatic changes as needed in response. These positional considerations are consistent with earlier cited evidence that the ventral AI shows strongly preferential involvement during tasks that require social or emotional valuation (which we take to be akin to salience assessment); with the recent conclusion that the ventral AI appears to be "predominantly engaged in internal and bodily homeostatic regulation" ([68], p. 586); and with the fact that the ventral AI forms the center of Seeley's et al.'s [55] "salience network".

We contend that among the ventral AI's key functions are to contextualize the earlier salience assessments of the CNA through the use of Lamina I information; to initiate appropriate homeostatic and behavioral changes in response to these assessments (primarily in partnership with the ACC, as discussed below); and to make the contents of the first body order map that it helps to represent available to the right dorsal AI for re-contextualization, thereby allowing conscious behavioral control when required.

Voluminous evidence links the AI and the ACC in emotional processing and in core self level functioning [22]. In particular, the ACC is known to play a key role in generating motivational drive and behavioral initiative [52]. However, like the AI, the ACC is a highly heterogeneous structure [69]. Therefore, we will address the functions of the specific sector of the ACC with which the ventral AI has evolved to work most closely, namely, BA 24b.

Our contention that the ventral AI has a close functional partnership with BA $24 \mathrm{~b}$ is predicated on the existence of a special class of very large, spindle shaped cells called Von Economo neurons (VENs). Within the human brain, the highest concentration of VENs is found linking the ventral AI [70] and BA 24 [71,72] (see Figure 6). Human beings have by far the highest density of VENs of any species studied to date. Further, "within the hominoid species, the group comprising humans and apes, the density of spindle cells declines with approximately the phylogenic distance from humans" [73] (p. 335).

The existence of VENs supports our contention that there is a strong link between the ventral AI and BA24b/ BA10r region whose connectivity was shown in Seeley's et al.'s [55] "salience network". Two functions that have been ascribed to this latter region are of particular importance for emotional processing, psychotherapy, and high level experiencing: the pre-conscious resolution of emotional conflicts and the assessment of the expected utility of possible actions.

In regard to the pre-conscious resolution of emotional conflicts, work studying conflict resolution in a sample of healthy participants is relevant [74]. This fMRI-based study involved two tasks. The first was a traditional cognitive Stroop task. The second was a modified emotional Stroop task. Successful conflict resolution during the 


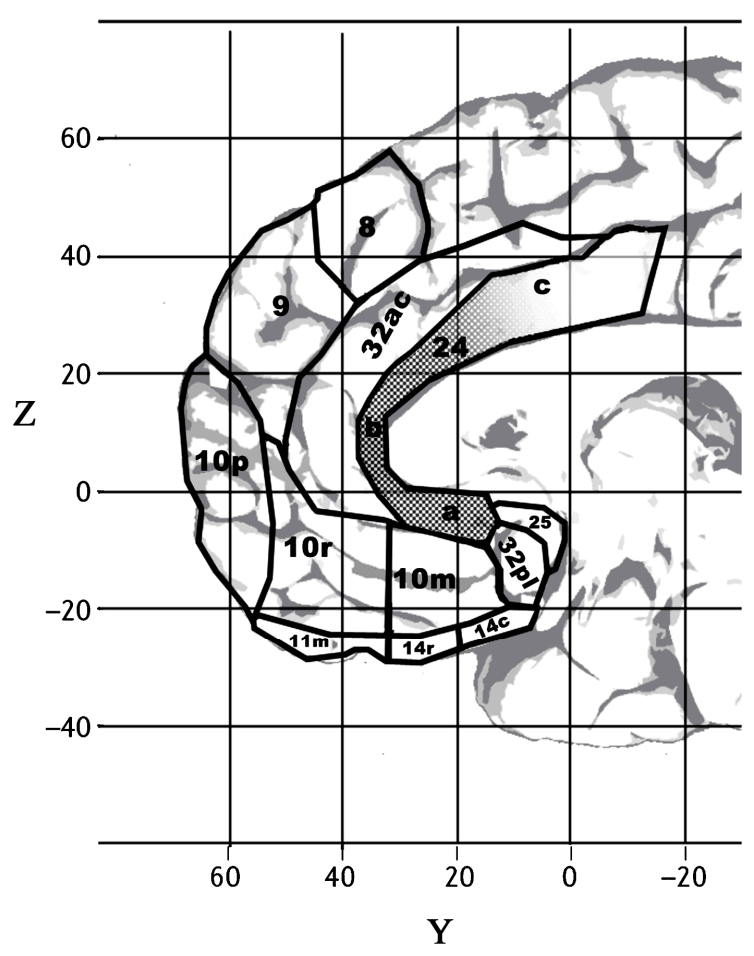

Figure 6. Von Economo neurons. As illustrated with the halftone gradient in this figure, which is labeled with Brodmann's area numbers, the main concentration of Von Economo neurons in the human brain is found in BA24, with a decreasing density moving dorsally [70].

cognitive Stroop test was associated with activity in the dlPFC (centered at 38,16,54), the core component of our evolutionarily newer cold cognition system. On the other hand, successful conflict resolution during the emotional Stroop test was associated with activity in posterior BA 10r $(-12,44,-2)$. Using the same experimental paradigm, research has also shown that this region achieves resolution of emotional conflict by down-regulating activation of the CNA [38], and the described form of emotion regulation is implicit, occurring beneath conscious awareness [75].

While the orbital and ventromedial prefrontal cortices (parts of the evolutionarily new hot cognition system) are crucial to assessing the expected utility value of possible rewards, they do not judge the expected utility value of possible actions, a role that has been ascribed to BA 10r [47], which we now discuss.

\subsubsection{Newer Mediation Subsystem}

We believe that the evolutionarily newer mediation network supports high level experiencing. Although this process is supported by many different posterior cortical regions, for purposes of clarity of exposition we focus here on the most important roles played by BA 10r and BA32a.

In addition to making their output accessible to right dorsal AI, evidence suggests that the bilateral ventral AIs also feed their output directly into anterior BA 10r [55]. Once in anterior BA 10r, this information derived from Lamina $\mathrm{I}$ is integrated into a single meta-representation of the body's overall homeostatic state. This meta-representation helps BA 10r to resolve salience-oriented problems that are beyond the processing capacities of the evolutionarily older mediation system. In other words, the bilateral ventral anterior insula and anterior BA 10r are the penultimate and ultimate convergence zones in a processing hierarchy that is primarily devoted to establishing the homeostatic meaning of stimuli and to resolving conflicts between different meaningful stimuli.

As with the ventral AI, BA 10r's salience related functions are usually performed outside of focal awareness. However, unlike the ventral AI representations, attention can be focused onto this BA 10r meta-representation in such a way that it can emerge fully into focal awareness [25]. We suggest that this requires the engagement of BA $32 \mathrm{ac}$. BA $32 \mathrm{ac}$ is not only involved in attentional control processes [76], it is also paired with BA 10r in the resting state network under consideration here [37] (see Figure 2(c)). Gaining conscious access to this meta-representation means that BA 10r's salience related processing can be performed under the guidance of conscious control. In short, we hold that this class of background feeling equates with a felt sense, and that the process of consciously working with this background feeling equates with high level experiencing.

We will offer a detailed neurally grounded conceptualization of high level experiencing in the next section. At this stage we will offer selected connectivity, phylogenic, and functional evidence to support the plausibility of our basic claim that BAs 10r and 32ac can support a felt sense type, background feeling as described by Gendlin.

In terms of connectivity, support for our model comes from evidence showing that the ventral $\mathrm{AI}$ is functionally connected to BA 10r and 32ac [54] (see Figure 4) and the finding of strong resting state linkages between bilateral, agranular/ventral AI and the BA 10r (peak at -24 , $56,10)[55]$.

Phylogenetically, BA 10 has distinctions that suggest that its role in human neural processing may be distinct from its role in other primates. Humans have by far the largest BA 10 of any hominoid species. BA 10 is proportionally much larger within the human brain than it is in the macaque brain. BA 10 may be the only area of prefrontal cortex that is much larger in human beings than it is in other primates $[73,77]$. Phylogenetic evidence also suggests that the other putative core component of the evolutionarily newer mediation system, BA $32 \mathrm{ac}$, is also very recent and relatively much more developed in human beings. For example, macaques have only one BA 
32 , while humans have evolved two functionally distinct BA 32s [78], one section (32ac/anterior cingulate) dorsal to the other (32pl/paralimbic). Both regions are marked on Figures 1, 2, 6 and 7.

Functionally oriented research also offers support for the role that we ascribe to BA 10r. In a meta-analysis considering the results of 104 neuroimaging studies reporting activation in BA 10 [79], BAs $10 \mathrm{r} / \mathrm{p}$ showed a very specific pattern of activation, only preferentially activating during tasks that involved both mentalizing and emotion. The average peak for these tasks centered in a focused area with average [SD] peak coordinates: $\mathrm{x}:-3$ [6.8]; y: 53.5 [3.9]; and y: 9.3 [9.5]). These findings suggest that the BA 10 region in question is preferentially involved in embodied forms of mentalizing, exactly as would be expected if it supported the consciously accessible, felt sense type representation that we posit it does.

Further work by Rolls and his colleagues offers support for another aspect of the functional role that we ascribe to BA 10r. Recall that during high level experiencing, behavior is guided through both continuous scale feelings of rightness and wrongness (as in mid level experiencing) and by binary, yes/no "felt give" responses. In this regard, there is strong significance to Rolls' claims [47] that the OFC-vmPFC based stimulus evaluation system is only capable of directing goal related activity as long as this can be achieved on a continuous rating scale basis. However, as Rolls notes, it is also

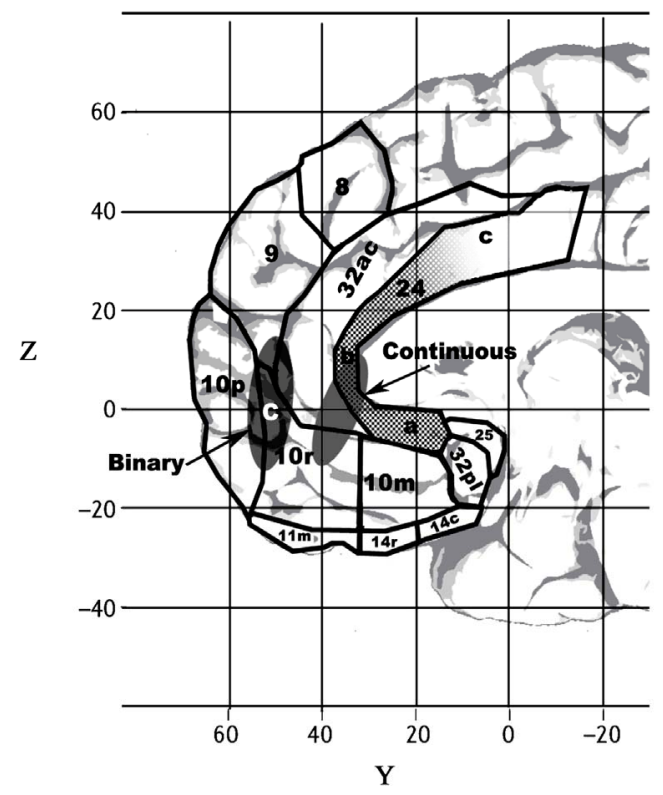

Figure 7. Regions associated by Rolls (2009) with continuous and binary decision making. Note that the region associated with binary decision-making is centered precisely on the common region for self-related processing networks found by both Andrews-Hanna et al. [36] and Liao et al. [37] (marked in both Figure 2 and this diagram with the letter “c”). See also Figure 3. sometimes necessary to make binary decisions and that this cannot be effectively achieved with a continuous scale. When a binary (choice) decision must be reached an additional tier of decision-making must be performed through the activation of a neural system "... that does not continuously represent the affective value of the stimulus, but which instead falls into a binary state..." [with]... the settling of an attractor network into one of its two... attractor states each representing a choice..." [47] (p. 235). Rolls contends that these higher order, binary decisions are made in the anterior medial BA 10r region. One study that Rolls uses to substantiate his claim about the primary role of BA 10r in making binary decisions is an fMRI study that exposed a sample of healthy participants to water at varying degrees of warmth [80]. During half of the trials participants were asked to rate the pleasantness of the stimuli on a continuous scale (e.g., from very pleasant to very unpleasant). During the other half of the trials participants were asked to make a binary, Yes/No decision as to whether or not they would want to have each stimulus repeated. Across all trials a region spanning the posterior ACC (BA 24b), the inferior area $32 \mathrm{ac}$, and posterior 10r (which we will refer to as 'the continuous rating region') tracked the subjective pleasantness (or reward value) of the stimuli in a continuous manner (see Figure 7), regardless of whether it was a "decide" or "rate trial". An anterior medial BA 10r region and the ventral anterior insula were the only two brain regions to preferentially activate during the Yes/No decision trials, as compared to the rating trials. This region precisely overlaps with the region identified in as the common region for the different self-related processing networks [36,37] (see Figures 2 and 7). This study also offers support for two other key claims. The first is that that the there is a posterior medial region that uses continuous scale rating to guide behavior, consistent with our assignation of this region a role in supporting continuous scale feelings of rightness and wrongness. The second is that there is a key functional hierarchical relationship between the bilateral ventral AI and anterior BA 10r.

Another aspect of Rolls' model [47] supports the contention that this brain region performs binary decisions. Rolls points out that human beings have a broad diversity of homeostatic needs (e.g. food, shelter, social contact), all of which are active to greater and lesser extents at any one time. It would be very inefficient or impossible to attempt to satisfy all of our homeostatic needs all at once. $\mathrm{He}$ argues that the brain must therefore support ongoing meta-representations of all of our basic needs as domains. He identifies the orbital medial PFC (BA 11) as the key area for supporting these kinds of meta-representations (example peak: BA 11;2, 52,-18). Within this orbital medial $\mathrm{PFC}$ region there is an ongoing neural competi- 
tion between these domain meta-representations [80]. This allows whichever need domain is the most salient to be selected for preferential processing by the $\mathrm{mOFC}$ and vmPFC.

Rolls goes on to claim that when there is equal activity between two or more goal domain meta-representations, the medial orbital frontal cortex (BA 11) calls on the anterior medial BA 10r to resolve the conflict [47]. BA 10r achieves this by allowing the relevant domains to directly compete with each other in a binary fashion until one "wins", thereby allowing the attractor landscape to settle into a stable basis of attraction. In turn, this settling down of the attractor landscape allows the winning domain to send its current needs into the previously described continuous rating system so that these needs can begin to be addressed in a focused, efficient manner. This resolving of between-domain conflicts has strong relevance for psychotherapy, and particularly for high level experiencing. We will return to this issue at the end of the article.

\subsection{The Neurology of Experiencing}

We will now draw on the ideas expressed thus far to present portraits of how we believe low, medium, and high level experiencing are instantiated in the brain.

\subsubsection{Low Level Experiencing}

Our conceptualization of low level experiencing is analogous to Damasio's Somatic Marker Hypothesis (SMH) that was alluded to earlier [23]. We will begin by summarizing the SMH before interpreting it specifically from the perspective of our neural model.

The SMH is based on the contention that when similar kinds of stimuli are experienced, similar body state/cognitive processing changes are enacted in response. When a particular stimulus has been paired consistently enough with a particular set of internal changes (especially in early development) that stimulus can automatically and unconsciously cause the nervous system to generate the internal changes that have become associated with it [21, 23].

Damasio argues that, during our lives, each human being develops an individualized palate of these automatic reactions, which he refers to as "secondary emotional responses", and suggests that reasoning can be understood as the process of sequentially making a series of related decisions. In line with the traditional understanding of decision-making, he argues that when we make a decision we mentally generate a range of response/outcome scenarios. However, before those scenarios are processed through a laborious cost/benefit analysis, they are evaluated through the use of secondary emotions. The imagined outcomes in scenarios that produce negative "secondary emotions" are "somatically marked" by the $\mathrm{mOFC} / \mathrm{vmPFC}$ subsystem as being negative [48]. As Da- masio says "... when the bad outcome connected with a given response option comes to mind, however fleetingly, you experience an unpleasant... response in your... gut..." [23] (p. 173). These possible outcomes can be quickly rejected, often beneath conscious awareness. Conversely, the imagined outcomes that produce pleasant secondary emotions are marked as being worthy of conceptual cost/benefit analysis. The use of fast acting "hot cognition" in this way allows us to focus the operation of our limited "cold" cognitive resources on considering a manageable number of options during cost/benefit analysis.

In line with the SMH, low level experiencing essentially involves operation of the cold cognition system operating under the (often covert) biasing operations of "somatic markers" generated by the hot cognition system [24]. In this mode, the mediation system is relatively unactivated and running entirely in the background.

A final contention around the neural dynamics of low level experiencing emerges in response to two factors. The first involves the finding that, in a phenomenological state analogous to low level experiencing, the mOFC (centered in BA 11 at $-4,56,-24$ ) showed very strong connectivity with the right anterior insula [81]. This suggests that, in this phenomenological mode, mOFC continually references the body maps in the right anterior insula so that it can evaluate whether encountered stimuli should be viewed as "being good or bad... [for] the "self" [81] (p. 8). The second factor involves our contention that during low level experiencing top down input into the right dorsal AI is stronger then Lamina I input. In combination, these two factors lead us to the following contention: that during low level experiencing the mOFC/ vmPFC subsystem performs the two functions that are of primary relevance to our model (somatic marker generation and regulation of the amgdala's CNA via the BLA) while using a largely simulated touchstone with which to ground its assessments and reactions (see Figure 8).

\subsubsection{Mid Level Experiencing}

Our conceptualization of mid level experiencing involves two key alterations from the scenario described above (Figure 9). First, there is a lessening of top down input from the evolutionarily newer cold cognitive system into the dorsal AI. This lowers the dorsal AI's level of overall activation. Reduced top down input also allows authentic, real time, Lamina I derived information to more strongly influence the right dorsal AI's computations. These changes slow the process of salience assessment. However, they also bring these assessments, along with the associated activity of the mOFC/vmPFC system [81], more closely into line with the current homeostatic contexts. The second key alteration is that the evolutionarily older mediation network is now more strongly engaged. 
The ventral $\mathrm{AI}$ and the $\mathrm{mPFC}$ based continuous rating region (informed by Lamina I input) can now participate more strongly during salience assessment and during the resolution of emotional conflicts [38]. The continuous scale feelings of rightness and wrongness that these regions generate begin to operate in parallel with the cold cognition/somatic marker based mechanisms. Reference to these emergent feelings of rightness and wrongness becomes an alternate means of guiding both cold cognition and behavior.

\subsubsection{High Level Experiencing}

In order to invite a state of high level experiencing, the client intentionally generates a mental image of the life situation that she wishes to address as a whole, as if she is mentally standing back to look at large mural of the overall situation [27]. While holding this image in her mind's eye, the client focuses on the background feeling that gradually emerges in response to this mental image, typically taking between 90 and 120 seconds. The therapist asks guiding questions in order to draw out the most salient elements that are implicitly contained within it. Once these elements have been drawn out, they can then be re-symbolized within the evolutionarily newer cold cognition system, so that they can help to guide the conscious reasoning process.

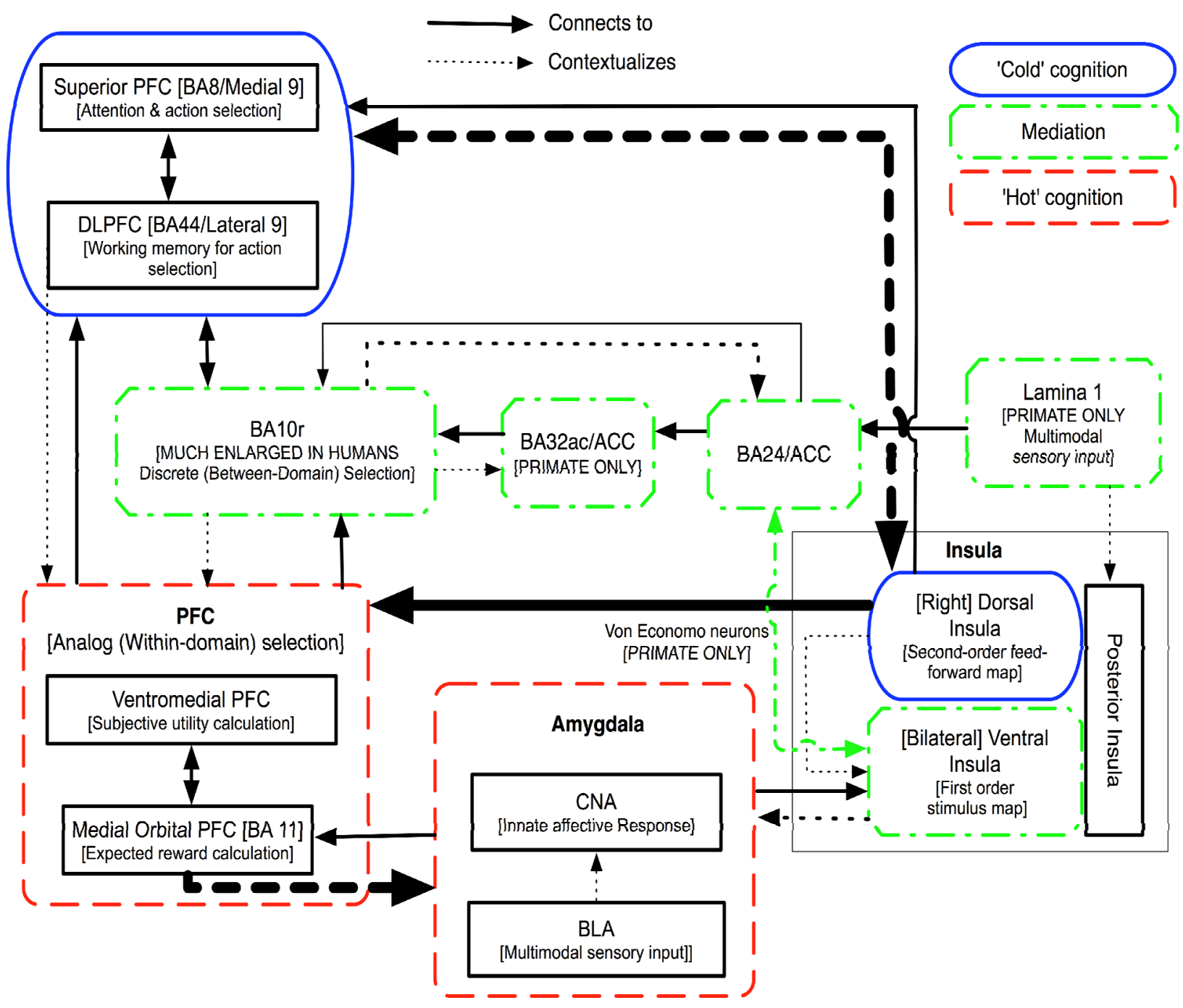

Figure 8. Low Level Experiencing. Solid pathways indicate neurological connectivity. Dotted arrows indicate "contextualization": i.e. that the content and output of the recipient region has valence and/or meaning that is modified by input from the source region. The main pathways implicated in Low Level processing are shown with bold lines. Because of the strong emphasis on top-down regulation of the dorsal insula, the PFC "hot cognition" system functions in a "simulated environment", with the PFC regulating the innate emotional response system not based on real world information, so much as on stored ideas about that world. The mediation system is "locked out" by the stronger top down processes, and thus has relatively little influence. 


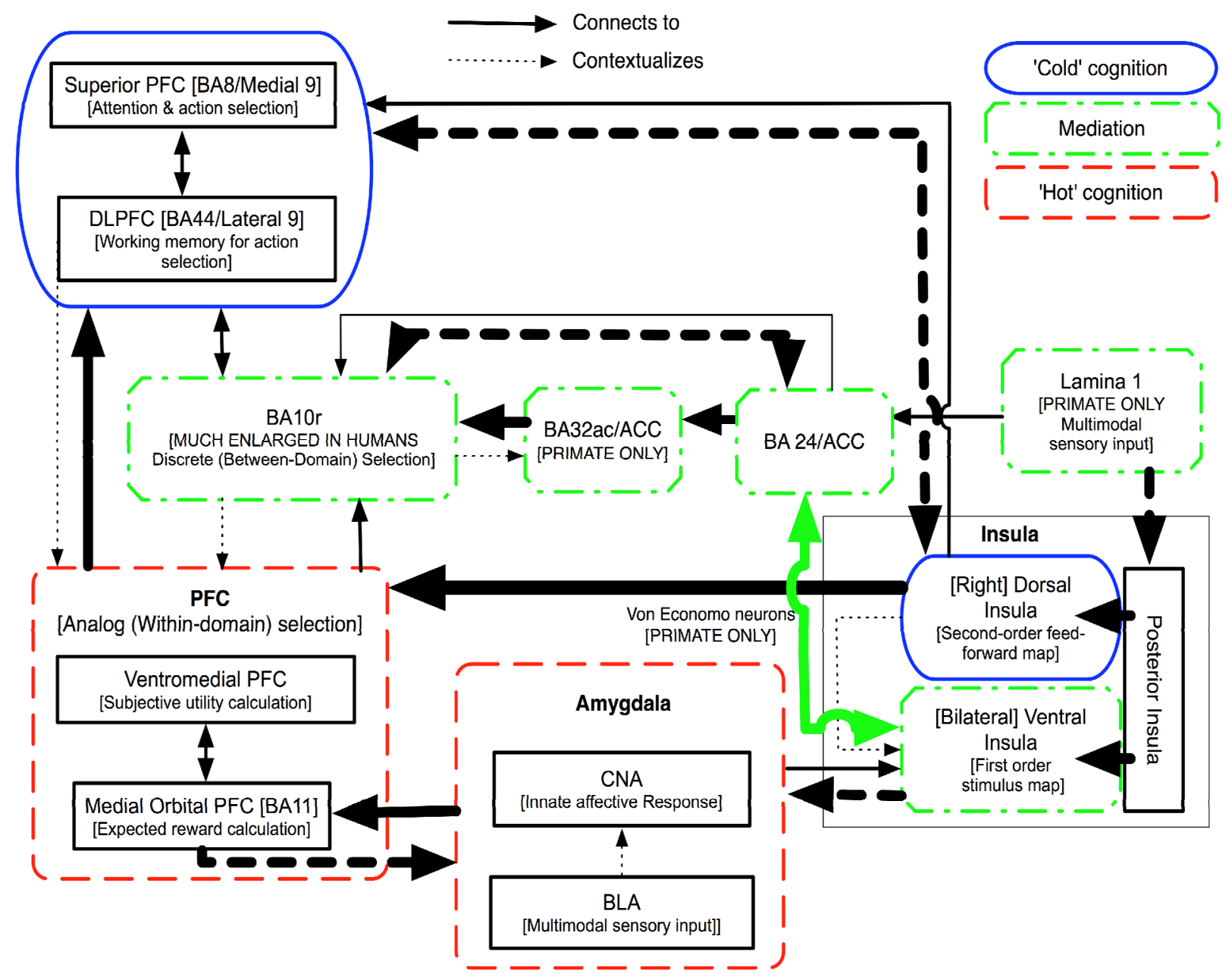

Figure 9. Mid-Level Experiencing. The main pathways implicated in Mid Level Experiencing are shown with bold lines. Solid pathways indicate neurological connectivity. Dotted arrows indicate "contextualization": i.e. that the content and output of the recipient region has valence and/or meaning that is modified by input from the source region. The main pathways implicated in Mid Level processing are shown with bold lines. The influence of top-down "simulated" input is weaker than in Low Level Experiencing, allowing a more salient role for the first-order body map of current homeostatic states in ventral anterior insula. The ventral anterior insula now more strongly generates authentic body maps and (in concert with the ACC node of the older mediation network) also produces continuous scale feelings of rightness/wrongness that can influence the cold cognition system (operating in parallel) in a bottom-up way.

We now offer a detailed description of how we believe this process is neurally instantiated (see Figure 10). First, the construction of a "mental mural" of the overall situation activates relevant memories. The posterior parietal cortex plays a key role in this memory related processing (see Figure 1). Next, the hot and cold cognition systems work together to reprocess these memories into a consciously accessible mental scene. Attention to this mental mural stimulates changes within the body proper [23]. These somatic changes are then read back by Lamina I and strongly represented in the ventral AI.

At this stage during high level experiencing, BA 32ac focuses conscious attention onto the anterior BA 10r region, where Lamina I derived information is represented into a single meta-representation. As a result, the felt sense of the overall situation emerges into conscious awareness. The tremendous richness of the homeostatic information being fed forward from Lamina I means that this background feeling can only be consciously represented at a low resolution, accounting for the inchoate, ephemeral quality of a felt sense.

Once the felt sense has formed in this way, the use of guiding questions (such as "What is this worst part of this whole situation?" [27]) intensifies the most salient possibility from among the tremendous number of possibilities that are weakly activated by considering the relevant life situation and that are all therefore, implicitly, part of the background feeling. When that possibility is intensified, the activity of its associated somatic profile also intensifies automatically. Information about the so- 
matic profile of the dominant activated representation is captured by Lamina I and can then represented in the ventral AI body maps.

When the most salient possibility in question is strongly activated, its associated somatic profile will stand out from the background of the felt sense. For example, a somatic sensation such as a tightness in the throat will often begin to emerge from the client's background awareness. When this occurs, the client is encouraged to shift her focal attention from the background felt sense to the physical sensation. This focus can eventually trigger the emergence of an associated conscious "mental image" within the evolutionarily new cold cognition system. Such a "mental image" [23] most commonly emerges in the form of a relevant visual image, memory, and/or focal emotion. In other words, in high level experiencing the brain to body relationship is worked in reverse: fo- cusing attention onto the somatic profile has been used as a means of making a mental image more salient so it can be conceptualized and communicated. During this process, the rightness of each emergent mental image can be confirmed by proposing a binary checking statement and awaiting a felt give response.

\section{Implications of the Model for Conceptualizations of Psychopathology}

The tri-partite model presented here allows for a useful re-conceptualization of psychopathology as an overly rigid, inflexible relationship between the three subsystems we have described $[82,83]$. In order to communicate this over-all conceptualization, we will now use our model to conceptualize three distinct routes into psychopathology: through under- or over-regulation of emotion, or through an impaired high experiencing ability.

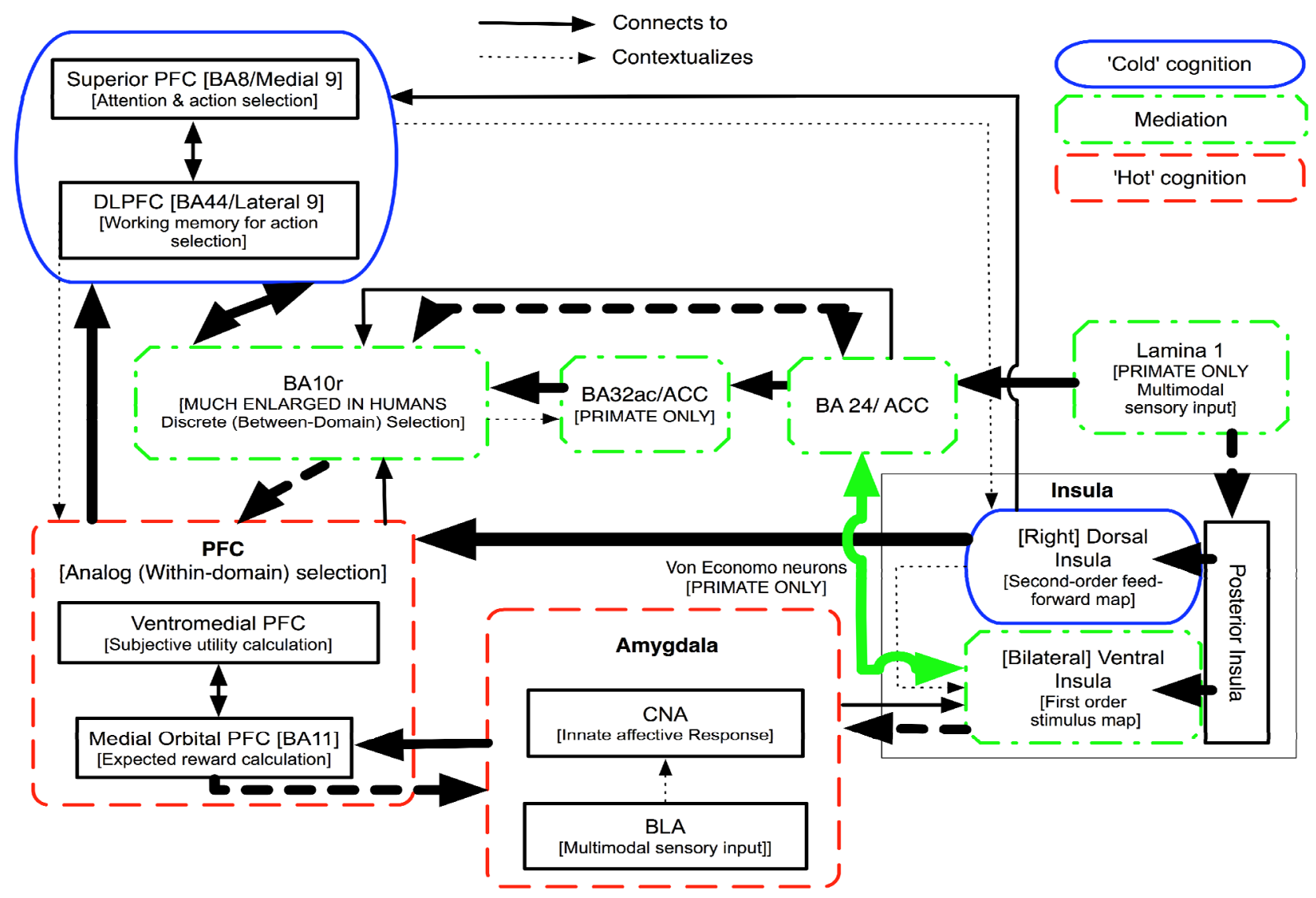

Figure 10. High-Level Experiencing. The main pathways implicated in High Level Experiencing are shown with a bold line. Solid pathways indicate neurological connectivity. Dotted arrows indicate 'contextualization': i.e. that the content and output of the recipient region has valence and/or meaning that is modified by input from the source region. The main pathways implicated in High Level processing are shown with bold lines. The main characteristic of High Level Experiencing is the activation of the recent, human-only mediation system, and the consequent ability to provide the cold cognition system with a much richer, more fluid, and more consciously accessible (though still inchoate) homeostatic representation of the present situation than is possible in Mid or Low Experiencing. Lamina I input from the body mapping provides an additional meta-e-presentation that is more directly accessible to consciousness than the insula maps, and that has "privileged access" to BA10r. BA10r has the potential to use this somatic mapping information to "shift the perspective" of the whole system, bringing new possibilities into play rather than limiting the system to a single dimension of analysis. 


\subsection{Problems of Emotional over and/or under Regulation}

We will begin by addressing two linked forms of psychopathology that typically lead to more severe dysfunction and thus to more frequent DSM diagnoses. The first occurs when people become stuck in a "conceptualizing/doing" [82] mode in which cold cognition systems are over-dominant. These people lose touch with their innate emotional responses and action tendencies and tend to experience symptoms related to the experiential emptiness caused by living as ideas rather than as embodied processes. Diagnoses of Generalized Anxiety Disorder and Dysthymia are perhaps most typical for such people.

The second of these basic routes into psychopathology appears when people lack the self-regulation abilities necessary to avoid becoming stuck in a "mindless emoting" [82] mode of processing. People who are stuck in this mode suffer the interpersonal and intrapersonal effects of living in a state of unregulated impulsivity and emotional reactivity. Because they cannot regulate their innate emotional responses, they experience the self as something that happens to them, rather than as an integrated guided system. Diagnoses of the impulse control and substance abuse related disorders are perhaps most typical.

We believe that, despite appearances to the contrary, these apparently opposing forms of psychological dysfunction stem from a common cause: that people are living their lives rigidly stuck in a low level of experiencing. This leads to a maladaptive, systemic dominance of the evolutionarily new cold cognition system over the mediation system. We believe that this has deleterious effects on psychological health for two basic reasons.

First, in low level experiencing, the top down, "as-if" body loop is strongly engaged. We contend that this maladaptively up-regulates the right dorsal AI, with the effect that salience assessments flowing up from ventral AI will be brought more continually and more intensely into conscious awareness. This contention is consistent with findings that hyperactivity in the right dorsal AI (centered at 27, 22,3) has been associated with "Anxiety Sensitivity" [84], a fear of anxiety related sensations. Anxiety sensitivity is associated with a tendency to become hyper-vigilant toward one's embodied responses and is also a vulnerability factor for the development of clinically significant anxiety and mood disorders [85].

Second, continual engagement of the "as-if-bodyloop" means that cold cognition is no longer strongly constrained by a core self embedded within a particular place and time. Further, over time a stimulus can become paired with particular as-if-body-loop simulations so that encountering that stimulus automatically triggers the associated simulated sense of self-in-world [23]. We be- lieve that this is a critical factor for the development of psychopathology because it can lead to the generation of what have referred to as "maladaptive core schemes" [23]. Maladaptive core schemes are typically based in shame or fear, often involving a background sense of the self as bad or weak [86]. Maladaptive core schemes can be clinically distinguished from generative (even if possibly aversive) real experiential states because they have a stale, unchanging quality, a quality of stuckness [28]. Ultimately, clinically diagnosable psychopathology emerges when a maladaptive core scheme of this kind becomes automatically paired to the activation of a basic, adaptive (typically attachment related) need [28].

Problems of emotional over-control and under-control both arise from a maladaptive dominance of the evolutionarily new cold cognition system. We believe that as long as this client is only capable of meeting his needs in at low level of experiencing that he will remain "gridlocked" [82]: his evolutionarily new cold and hot cognition systems will continue jointly over-controlling the adaptive output of his evolutionarily old mediation network (as manifest in innate emotional responses such as his adaptive sadness at social isolation), rendering him incapable of self organizing in such a way that he can effectively meet his homeostatic needs. A clinically diagnosable psychopathology is likely to be the end result.

Figure 9 illustrates how we believe this client's psychopathology could be ameliorated if he becomes capable of fulfilling his attachment needs while in a mid level of experiencing. For reasons that were described earlier, processing his attachment related needs in this more present focused neural mode should lead to: lessened exaggeration of potentially threatening threat related social cues; lessened hyper-awareness of the emotions that arise in response to potentially threatening social cues; an improved ability to use feelings of rightness and wrongness to guide his approach behaviour; and improved emotional conflict resolution abilities. In turn, these changes should help him overcome experiential avoidance by expressing his attachment related action tendencies despite the continued presence of now moderated levels of fear and arousal [87]. In turn, the accrual of positive attachment related experiences gained during these interactions should gradually allow him to begin linking the emergence of his adaptive, attachment related emotions to new, positively valenced memories. Over time, this co-activation of his attachment related emotions and these new memories should gradually lessen the psychopathological influence of his maladaptive core scheme [28].

\subsection{Psychopathology Emerging from Impaired High Experiencing Ability}

The final form of dysfunction that we will consider from 
the perspective of our model involves a subtler, more common psychopathology, in which clients who have well functioning evolutionarily older mediation systems are unable to use their evolutionarily new mediation system effectively enough to enter high level experiencing when it would be adaptive to do so. People with this processing pattern will tend to remain psychologically functional and will receive DSM diagnoses less frequently. However, they will also tend to experience distress at points in their lives when two valued goal domains come strongly, and typically implicitly, into conflict [49] because they will struggle to consciously identify the true source of their stuckness. This deficit impairs their abilities to resolve these conflicts in a flexible and self-compassionate way, leaving those who suffer from it feeling depleted and less fulfilled than, on the surface, it seems they "should".

\section{Support for the Model of Psychopathology}

Support for this conceptualization of psychopathology comes from neuroimaging studies of both neuroticism generally, and more specifically from studies of anxiety and depression

\subsection{Neuroimaging Research into Neuroticism}

The personality construct of neuroticism can be defined as "a trait disposition to experience Negative Affect" [88] (p. 1126). In a 2-year longitudinal study with a sample of over 600 outpatients who received treatment for MDD, GAD, or Social Phobia "... all of the temporal covariance of the DSM-IV disorder constructs was accounted for by change in... [neuroticism scores]" [89] (p. 269; emphasis added). These results point toward a pair of key conclusions. First, neuroticism may be at the very heart of much psychological dysfunction $[88,89]$. Second, neuroticism "... may be therapeutically malleable, and that this in fact mediates the extent of change in the emotional disorders ..." [89] (p. 263).

In this light, it becomes significant that neuroimaging studies involving measures of neuroticism are consistent with our model of psychopathology. An fMRI experiment involving a sample of healthy participants engaging in a gambling task involved choosing between risky or safe gambling choices [90]. The risky bets involved the possibility of larger payouts but also of being punished with large losses. This study produced a pair of key findings. First, degree of activation in the right dorsal AI following a punished risky choice correlated significantly $(r=0.72)$ with NEO neuroticism scores. Second, degree of right dorsal AI activity (centered at 32, 18, 7) following a punished risky choice predicted the likelihood that the participant would opt for a safe gamble on the following trial. In other words, the more neurotic a partici- pant was the more they activated the right dorsal AI during a negative experience, and the more likely they were to subsequently let a desire to avoid that experience inform their subsequent behavior, as occurs in the development of affect phobia.

Using PET, it has been found that neuroticism is negatively correlated with resting state metabolism $(\mathrm{r}=-0.63)$ levels in the dorsal, posterior insula $(-32,-22,16)[91]$ : that is in Lamina I's entry point into the insula [51]. This is consistent with our claim that a hallmark of psychopathology is a tonic under-representation of Lamina I input, relative to top down input, into the insula.

An fMRI study using affective pictures found that immediately following the presentation of angry and fearful faces, neuroticism was negatively correlated with connectivity between BA $24 \mathrm{~b}(12,36,12$, in the continuous rating region) and the CNA $(\mathrm{p}<0.01)$ [92]. This is consistent with our contention that psychopathology is associated with weakened functional integrity within the mediation network, and with reduced abilities of the ventral $\mathrm{AI}$ and the continuous rating region to regulate the CNA adequately during salience assessment.

Finally, another PET study has shown that neuroticism inversely correlates with resting state metabolism in a BA 10/32ac centered cluster [93]. This is consistent with our contention that weakened functional integrity within the mediation network is strongly associated with psychopathology. This finding also offers support for our inclusion of BA 10/32ac as a component of the mediation network.

\subsection{Neuroimaging Research into Anxiety and Depression}

Depression has been associated with dmPFC hyperactivity [94]. Alternately, resting state levels of activity in the BA $24 \mathrm{~b}$ have recently found to be negatively correlated with anhedonia scores [95]. Increased resting state levels of activity in the same region have also repeatedly been found to predict improved outcome response in the treatment of depression [96,97].

Hyperactivity in the dmPFC has also recently been associated with anxiety arising from a sense of social threat [98]. Finally, it has been found that there is a positive association between left mOFC volume and worry scores in a cohort of older adults with Generalized Anxiety Disorder (GAD) [99], supporting a model of GAD as rooted in maladaptive, frontal over-control of limbic activity. In GAD, this may occur largely through the use of worry, putatively associated with medial OFC activity, as a maladaptive means of down-regulating the CNA (via the BLA).

\section{Conclusion}

Emotion has made a resurgence in psychotherapeutic 
theory and practice in recent years. Partly through the advent of neuroimaging, there has been a growing appreciation of the profoundly important roles played by emotional processes in both sustaining health and in engendering dysfunction. However, during these advances, emotion has been almost exclusively understood as focal emotion. We suggest that the time is now ripe for the field to begin appreciating, both theoretically and clinically, the distinctions that we have highlighted between cold cognition and different classes of emotion.

Cold cognition, somatic markers, focal emotion, feelings of rightness/wrongness, and felt senses/felt gives all appear to play vital roles in human information processing. However, based on the model we have presented, we believe that ameliorating psychopathology essentially involves helping clients to strengthen their mediation systems. This, in turn, involves helping them strengthen their abilities to make adaptive use of the later three classes of response. For this to occur, clinicians need to capable of assessing when the client is unproductively stuck in low level experiencing; of helping the client deepen into a more productive medium level experiencing; of assessing when the client has been successfully processing at a medium level of experiencing for some time yet has still failed to find a "right" next step forward (typically because of implicit, between goal-domain conflict); and finally, of helping the client get "unstuck" if she does fail in this way, by helping her to shift into high level experiencing until the elusive next step has emerged.

Eugene Gendlin [27] has written extensively about how the value of a clinician's ability to assess and help deepen a client's level of experiencing transcends any particular psychotherapeutic model. Experiencing has "... been shown to be predictive of good treatment outcome across most major schools of psychotherapy, including client-centered therapy, CBT, psychodynamic therapy, and emotion-focused psychotherapy." (p. 117). Because of its essential role in psychotherapy, training student therapists how to work skillfully with the experiencing variable, a trainable skill [12], should be as ubiquitous in training programs as teaching students how to form positive therapeutic alliances with their clients.

In this paper we have grounded the ideas of Gendlin in neural processes. Our hope is that the effort to begin putting the modes of cognition he has described on a sound neurological basis will have indirect benefits to the psychotherapist, by situating a process that seems to be arational in sound empirical science that is accessible to rational cold cognition. When we understand how our very structure forces us to live as a dynamic system in interplay between different modes of processing, it may be easier for us inclined too far to using one mode over the others to access the range of these modes in a skillful, flexible way that maximizes our behavioral adaptivity in our environment.

\section{REFERENCES}

[1] M. E. P. Seligman, "The Effectiveness of Psychotherapy: The Consumer Reports Study," American Psychologist, Vol. 50, No. 12, 1995, pp. 965-974. doi:10.1037/0003-066X.50.12.965

[2] C. M. Boisvert and D. Faust, "Leading Researchers' Consensus on Psychotherapy Findings: Implications for the Teaching and Conduct of Psycho-Therapy," Professional Psychology: Research and Practice, Vol. 34, No. 5, 2003, pp. 508-513. doi:10.1037/0735-7028.34.5.508

[3] S. Rosenzweig, "Some Implicit Factors in Diverse Methods of Psychotherapy," American Journal of Orthopsychiatry, Vol. 6, No. 3, 1936, pp. 412-415. doi:10.1111/j.1939-0025.1936.tb05248.x

[4] L. Luborsky, B. Singer and L. Luborsky, "Comparative Studies of Psychotherapies: Is It True That 'Everyone Has Won and All Must Have Prizes?'," Archives of General Psychiatry, Vol. 32, No. 8, 1975, pp. 995-1008. doi:10.1001/archpsyc.1975.01760260059004

[5] L. Carroll, "Alice in Wonderland," Macmillan and Co., London, 1865.

[6] L. A. Robinson, J. S. Berman and R. A Neimeyer, "Psychotherapy for the Treatment of Depression: A Comprehensive Review of Controlled Outcome Research," Psychological Bulletin, Vol. 108, No. 1, 1990, pp. 30-49. doi:10.1037/0033-2909.108.1.30

[7] M. L. Smith, G. V. Glass and T. I. Miller, "The Benefits of Psychotherapy," Johns Hopkins Press, Baltimore, 1980

[8] B. E. Wampold, G. W. Mondin, M. Moody, F. Stich, K. Benson and H. Ahn, "A Meta-Analysis of Outcome Studies Comparing Bona Fide Psychotherapies: Empirically, 'All Must Have Prizes'," Psychological Bulletin, Vol. 122, No. 3, 1997, pp. 203-215. doi:10.1037/0033-2909.122.3.203

[9] L. Luborsky, "Are Common Factors across Different PsyChotherapies the Main Explanation for the Dodo Bird Verdict That 'Everyone Has Won and All Must Have Prizes'?" Clinical Psychology: Science and Practice, Vol. 2, No. 1, 1995, pp. 106-109. doi:10.1111/j.1468-2850.1995.tb00033.x

[10] L. Luborsky, L. Diguer, D. A. Seligman, R. Rosenthal, E. D. Krause, S. Johnson, G. Halperin, M. Bishop, J. S. Berman and E. Schweizer, "The Researcher's Own Therapy Allegiances: A 'Wild Card' in Comparisons of Treatment Efficacy," Clinical Psychology: Science and Practice, Vol. 6, No. 1, 1999, pp. 95-106. doi:10.1093/clipsy/6.1.95

[11] S. B. Messer and B. E. Wampold, "Let's Face the Facts: Common Factors Are More Potent than Specific Therapy Ingredients," Clinical Psychology: Science and Practice, Vol. 9, No. 1, 2002, pp. 21-25. doi:10.1093/clipsy.9.1.21

[12] M. N. Hendricks, "Focusing-Oriented/Experiential Psychotherapy," In: D. J. Cain and J. Seeman, Eds., Humanistic Psychotherapies: Handbook of Research and Practice, American Psychological Association, Washington 
DC, 2002, pp. 221-252. doi:10.1037/10439-007

[13] L. S. Greenberg, L. M. Korman and S. C. Paivio, "Emotion in Humanistic Therapy," In: D. Cain and J. Seeman, Eds., Handbook of Humanistic Psychotherapy, APA Press, Washington DC, 2002, pp. 499-530. doi:10.1037/10439-016

[14] M. H. Klein, P. Mathieu-Coughlan and D. J Kiesler, "The Experiencing Scales," In: L. Greenberg and W. M. Pinsoff, Eds., The Psychotherapy Process: A Research Handbook, Guilford, New York, 1986, pp. 21-72.

[15] W. Whelton, "Emotional Processes in Psychotherapy: Evidence across Therapeutic Modalities," Clinical Psychology \& Psychotherapy, Vol. 11, No. 1, 2004, pp. 5871. doi:10.1002/cpp.392

[16] M. H. Klein, P. L. Mathieu, E. T. Gendlin and D. J. Kiesler, "The Experiencing Scale: A Research and Training Manual," Vol. 1, University of Wisconsin Extension Bureau of Audiovisual Instruction, Madison, 1969.

[17] D. J. Kiesler, "The Process of Psychotherapy," Aldine, Chicago, 1973.

[18] A. Pascual-Leone, "Dynamic Emotional Processing in Experiential Therapy: Two Steps Forward, One Step Back," Journal of Consulting and Clinical Psychology, Vol. 77, No. 1, 2009, pp. 113-126. doi:10.1037/a0014488

[19] R. N. Goldman, "Theme-Related Depth of Experiencing and Change in Experiential Psychotherapy with Depressed Clients," York University, Toronto, 1997.

[20] E. T. Gendlin, "Experiencing and the Creation of Meaning," Northwest University Press, Evanston, 1997.

[21] R. Damasio, "The Feeling of What Happens: Body and Emotion in the Making of Consciousness," Harcourt Brace, New York, 1999.

[22] D. Craig, "How Do You Feel? Interoception: The Sense of the Physiological Condition of the Body," Nature Reviews Neuroscience, Vol. 3, 2002, pp. 655-666.

[23] A. R. Damasio, "Descartes' Error: Emotion, Reason and the Human Brain," G. P. Putnam's Sons, New York, 1994.

[24] V. Goel and R. J. Dolan, "Reciprocal Neural Response within Lateral and Ventral Medial Prefrontal Cortex during Hot and Cold Reasoning," NeuroImage, Vol. 20, No. 4, 2003, pp. 2314-2321. doi:10.1016/j.neuroimage.2003.07.027

[25] R. D. Lane and K. McRae, "Neural Substrates of Conscious Emotional Experience: A Cognitive Neuroscientific Perspective," In: M. Beauregard, Ed., Consciousness, Emotional Self-Regulation and the Brain, John Benjamins, Philadelphia, 2003, pp. 88-122.

[26] E. T. Gendlin, "The Client's Client: The Edge of Awareness," In: R. L. Levant and J. M. Shlien, Eds., ClientCentered Therapy and the Person-Centered Approach. New Directions in Theory, Research and Practice, Praeger, New York, 1984, pp. 76-107.

[27] E. T. Gendlin, "Focusing-Oriented Psychotherapy: A Manual of the Experiential Method," Guilford, New York, 1996.

[28] L. S. Greenberg, L. N. Rice and R. E. Elliot, "Facilitating
Emotional Change: The Moment by Moment Process," Guilford, New York, 1993.

[29] L. N. Rice, C. J. Koke, L. S. Greenberg and A. K. Wagstaff, "Manual for Client Vocal Quality," Vols. I \& II, York University, Counselling and Development Centre, Toronto, 1979.

[30] H. Wiseman and L. N. Rice, "Sequential Analysis of Therapist-Client Interaction during Change Events: A Task-Focused Approach," Journal of Consulting and Clinical Psychology, Vol. 57, No. 2, 1989, pp. 281-286. doi:10.1037/0022-006X.57.2.281

[31] R. Buckner, J. Andrews-Hanna and D. Schacter, "The Brain's Default Network: Anatomy, Function and Relevance to Disease," Annals of the New York Academy of Science, Vol. 1124, 2008, pp. 1-38. doi:10.1196/annals.1440.011

[32] L. S. Greenberg and W. Malcolm, "Resolving Unfinished Business: Relating Process to Outcome," Journal of Consulting and Clinical Psychology, Vol. 70, No. 2, 2002, pp. 406-416. doi:10.1037/0022-006X.70.2.406

[33] M. Merleau-Ponty, "Phenomenology of Perception," Routledge, London, 1962.

[34] R. Damasio, "Looking for Spinoza: Joy, Sorrow and the Feeling Brain,” Harcourt, New York, 2003.

[35] G. Northoff, A. Heinzel, M. De Greck, F. Bermphol, H. Dobrowolny and J. Panskeep, "Self-Referential Processing in Our Brain - A Meta-Analysis of Imaging Studies of the Self," NeuroImage, Vol. 31, No. 1, 2006, pp. 440457. doi:10.1016/j.neuroimage.2005.12.002

[36] J. R. Andrews-Hanna, J. S. Reidler, J. Sepulcre, R. Poulin, and R. L. Buckner, "Functional-Anatomic Fractionation of the Brain's Default Network," Neuron, Vol. 65, No. 4, 2010, pp. 550-562. doi:10.1016/j.neuron.2010.02.005

[37] W. Liao, D. Mantini, Z. Zhiqiang, P. Zhengyong, D. Jurong, G. Qiyong, Y. Yihong and C. Huafu, "Evaluating the Effective Connectivity of Resting State Networks," Biological Cybernetics, Vol. 102, No. 1, 2010, pp. 57-69. doi:10.1007/s00422-009-0350-5

[38] A. Etkin, T. Egner, D. M. Peraza, E. R. Kandel and J. Hirsch, "Resolving Emotional Conflict: A Role for the Rostral Anterior Cingulate Cortex in Modulating Activity in the Amygdala," Neuron, Vol. 51, No. 6, 2006, pp. 871-882. doi:10.1016/j.neuron.2006.07.029

[39] A. Bechara, H. Damasio and A. R. Damasio, "Emotion, Decision Making and the Orbitofrontal Cortex," Cerebral Cortex, Vol. 10, No. 3, 2000, pp. 295-307. doi:10.1093/cercor/10.3.295

[40] W. Balleine and S. Killcross, "Parallel Incentive Processing: An Integrated View of Amygdala Function," Trends in Neurosciences, Vol. 5, No. 5, 2006, pp. 272279. doi:10.1016/j.tins.2006.03.002

[41] A. Etkin, K. E. Keller, A. F. Schatzberg, V. Menon and M. D. Greicius, "Disrupted Amygdalar Subregion Functional Connectivity and Evidence for a Compensatory Network in Generalized Anxiety Disorder," Archives of General Psychiatry, Vol. 66, No. 12, 2009, pp. 13611372. doi:10.1001/archgenpsychiatry.2009.104

[42] D. Öngür and J. L. Price, "The Organization of Networks 
within the Orbital and Medial Prefrontal Cortex of Rats, Monkeys and Humans," Cerebral Cortex, Vol. 10, No. 30, 2000, pp. 206-219. doi:10.1093/cercor/10.3.206

[43] J. O’Doherty, M. L. Kringelbach, E. T. Rolls, J. Hornak and C. Andrews, "Abstract Reward and Punishment Representations in the Human Orbitofrontal Cortex," Nature Neuroscience, Vol. 4, 2001, pp. 95-102. doi: $10.1038 / 82959$

[44] E. T. Rolls, "The Orbitofrontal Cortex and Reward," Cerebral Cortex, Vol. 10, No. 3, 2000, pp. 284-294. doi:10.1093/cercor/10.3.284

[45] A. Bechara, H. Damasio, A. R. Damasio and G. P. Lee, "Different Contributions of the Human Amygdala and Ventromedial Prefrontal Cortex to Decision-Making," Journal of Neuroscience, Vol. 19, No. 13, 1999, pp. 5473-5481.

[46] G. Schoenbaum, A. A. Chiba and M. Gallagher, "Neural Encoding in Orbitofrontal Cortex and Basolateral Amygdala during Olfactory Discrimination Learning," Journal of Neuroscience, Vol. 19, No. 5, 1999, pp. 1876-1884.

[47] E. T. Rolls, "From Reward to Decision-Making: Neuronal and Computational Principles," In: J. C. Dreher and L. Trembley, Eds., The Handbook of Reward and Decision Making, Elsevier, New York, 2009, pp. 97-134 doi:10.1016/B978-0-12-374620-7.00005-4

[48] X. Li, Z. L. Lu, A. D'Argembeau, M. Ng and A. Bechara, "The Iowa Gambling Task in fMRI images," Human Brain Mapping, Vol. 31, No. 3, 2009, pp. 410-423. doi:10.1002/hbm.20875

[49] N. Naqvi, B. Shiv and A. Bechara, "The Role of Emotion in Decision Making: A Cognitive Neuroscience Perspective," Current Directions in Psychological Science, Vol. 15, No. 5, 2006, pp. 260-264. doi:10.1111/j.1467-8721.2006.00448.x

[50] D. Craig, "Human Feelings: Why Are Some More Aware than Others?" Trends in Cognitive Sciences, Vol. 8, No. 6, 2004, pp. 239-241. doi:10.1016/j.tics.2004.04.004

[51] A. D. Craig, "Interoception and Emotion: A Neuroanatomical Perspective," In: M. Lewis, J. M. Haviland-Jones and L. F. Barrett, Eds., Handbook of Emotion, Chapter 16, 2007, Guilford Press, New York, pp. 272-288.

[52] I. A. Strigo, A. N. Simmons, S. C. Matthews, A. D. Craig, M. P. Paulus, "Association of Major Depressive Disorder with Altered Functional Brain Response during Anticipation and Processing of Heat Pain," Archives of General Psychiatry, Vol. 65, No. 11, 2008, pp. 1275-1284.

[53] F. G. Flynn, D. F. Benson and A. Ardila, "Anatomy of the Insula Functional and Clinical Correlates," Aphasiology, Vol. 13, No. 1, 1999. pp. 55-78. doi: $10.1080 / 026870399402325$

[54] S. M. Nelson, N. U. F. Dosenbach, A. L. Cohen, B. L. Schlaggar and S. E. Petersen, "Role of the Anterior Insula in Task-Level Control and Focal Attention," Brain Structure and Function, Vol. 214, No. 5-6, 2010, pp. 669-680.

[55] W. W. Seeley, V. Menon, A. F. Schatzberg, J. Keller, G. H. Glover, H. Kenna, A. L. Reiss and M. D. Greicius, "Dissociable Intrinsic Connectivity Networks for Salience Processing and Executive Control," Journal of Neurosci- ence, Vol. 27, No. 9, 2007, pp. 2349-2356. doi:10.1523/JNEUROSCI.5587-06.2007

[56] F. Kurth, K. Zilles, P. T. Fox, A. R. Laird and S. B. Eickhoff, "A Link between the Systems: Functional Differentiation and Integration within the Human Insula Revealed by Meta-Analysis," Brain Structure and Function, Vol. 214, No. 5-6, 2010, pp. 519-534. doi:10.1007/s00429-010-0255-Z

[57] J. A. Coan, H. S. Schaefer and R. J. Davidson, "Lending a Hand: Social Regulation of the Neural Response to Threat," Psychological Science, Vol. 17, No. 12, 2006, pp. 1032-1039. doi:10.1111/j.1467-9280.2006.01832.x

[58] N. I. Eisenberger, M. D. Lieberman and K. D. Williams, "Does Rejection Hurt? An fMRI Study of Social Exclusion," Science, Vol. 302, No. 5643, 2003, pp. 290-292. doi:10.1126/science.1089134

[59] E. K. Miller and J. Cohen, "An Integrative Theory of Prefrontal Cortex Function," Annual Review of Neuroscience, Vol. 24, No. 1, 2001, pp. 167-202. doi:10.1146/annurev.neuro.24.1.167

[60] M. Petrides, "Lateral Frontal Cortical Contribution to Memory," The Neurosciences, Vol. 8, No. 1, 1996, pp. 57-63. doi:10.1006/smns.1996.0008

[61] S. Kapur, R. Rose, P. F. Liddle, R. B. Zipursky, G. M. Brown, D. Stuss, S. Houle and E. Tulving, "The Role of the Left Prefrontal Cortex in Verbal Processing: Semantic Processing or Willed Action," Neuroreport, Vol. 5, No. 16, 1994, pp. 2193-2196. doi:10.1097/00001756-199410270-00051

[62] M. I. Leon and M. N. Shadlen, "Effect of Expected Reward Magnitude on the Response of Neurons in the Dorsolateral Prefrontal Cortex of the Macaque," Neuron, Vol. 24, No. 2, 1999, pp. 415-425. doi:10.1016/S0896-6273(00)80854-5

[63] T. Deacon, "The Symbolic Species," Norton, New York, 1998.

[64] C. D. Frith, "The Role of the dlPFC in the Selection of Action, as Revealed by Functional Imaging," In: S. Monsell and J. Driver, Eds., Control of Cognitive Processes: Attention and Performance, Vol. 18, MIT Press, Cambridge, 2000, pp. 549-565.

[65] M. P. Paulus and M. S. Stein, “An Insular View of Anxiety," Biological Psychiatry, Vol. 60, No. 4, 2006, pp. 383-387. doi:10.1016/j.biopsych.2006.03.042

[66] H. Critchley, "Neural Mechanisms of Autonomic Affective, and Cognitive Integration," The Journal of Comparative Neurology, Vol. 493, No. 1, 2005, pp. 154-166. doi:10.1002/cne.20749

[67] T. D. Wager and L. Feldman Barrett, "From Affect to Control: Functional Specialization of the Insula in Motivation and Regulation," 2010.

[68] C. Lamm and T. Singer, "The Role of Anterior Insular Cortex in Social Emotions," Brain Structure and Function, Vol. 214, No. 5-6, 2010, pp. 579-591. doi:10.1007/s00429-010-0251-3

[69] T. Paus, "Primate Anterior Cingulate Cortex: Where Motor Control, Drive and Cognition Interface," Nature Re- 
views Neuroscience, Vol. 2, 2001, pp. 417-424. doi: $10.1038 / 35077500$

[70] J. M. Allman, N. A. Tetreault, A. Y. Hakeem, K. F. Manaye, K. Semendeferi, J. M. Erwin and V. Goubert, "The Von Economo Neurons in Frontoinsular and Anterior Cingulate Cortex in Great Apes and Humans," Brain Structure and Function, Vol. 214, No. 5-6, 2010, pp. 495517. doi:10.1007/s00429-010-0254-0

[71] J. M. Allman, K. K. Watson, N. A. Tetreault and A. Y. Hakeem, "Intuition and Autism: A Possible Role for Von Economo Neurons," Trends in Cognitive Science, Vol. 9, No. 8, 2005, pp. 367-373. doi:10.1016/j.tics.2005.06.008

[72] U. Frith and C. D. Frith, "Development and Neurophysiology of Mentalizing," Transactions of the Royal Society of London, Series B, Vol. 358, No. 1431, 2003, pp. 459473. doi:10.1098/rstb.2002.1218

[73] J. Allman, A. Hakeem and K. Watson, "Two Phylogenetic Specializations in the Human Brain," Neuroscientist, Vol. 8, No. 40, 2002, pp. 335-346. doi:10.1177/107385840200800409

[74] T. Egner, A. Etkin, S. Gale and J. Hirsch, "Dissociable Neural Systems Resolve Conflict from Emotional versus Non-Emotional Distracters," Cerebral Cortex, Vol. 18, No. 6, 2008, pp. 1475-1484. doi:10.1093/cercor/bhm179

[75] A. Etkin, K. E. Prater, F. Hoeft, V. Menon and A. F. Schatzberg, "Failure of Anterior Cingulated Activation and Connectivity with the Amygdala during Implicit Regulation of Emotional Processing in Generalized Anxiety Disorder," American Journal of Psychiatry Online Advance, Vol. 167, No. 5, 2010, pp. 545-554.

[76] B. Hölzel, U. Ott, H. Hempel, A. Hackl, K. Wolf, R. Stark and D. Vaitl, "Differential Engagement of Anterior Cingular and Adjacent Medial Frontal Cortex in Adept Meditators and Non-Meditators," Neuroscience Letters, Vol. 21, No. 1, 2007, pp. 16-21. doi:10.1016/i.neulet.2007.04.074

[77] K. Semendeferi, K. Teffer, D. P. Buxhoeveden, M. S. Park, S. Bludau, K. Amunts, K. Travis and J. Buckwalter, "Spatial Organization of Neurons in the Frontal Pole Sets Humans Apart from Great Apes," Cerebral Cortex, Vol. 21, No. 7, 2011, pp. 1485-1497. doi:10.1093/cercor/bhq191

[78] D. Öngür, A. T. Ferry and J. L. Price, "Architectonic Subdivision of the Human Orbital and Medial Prefrontal Cortex," The Journal of Comparative Neurology, Vol. 463, No. 3, 2003, pp. 425-449. doi:10.1002/cne.10609

[79] S. J. Gilbert, S. Spengler, J. S. Simons, J. A. Steele, S. M. Lawrie, C. D. Frith and P. W. Burgess, "Functional Specialization within Rostral Prefrontal Cortex (Area 10): A Meta-Analysis," Journal of Cognitive Neuroscience, Vol. 18, No. 6, 2006, pp. 932-948. doi:10.1162/jocn.2006.18.6.932

[80] F. Grabenhorst, E. T. Rolls and B. A. Parris, "From Affective Value to Decision-Making in the Prefrontal Cortex," European Journal of Neuroscience, Vol. 28, No. 9, 2008, pp. 1930-9. doi:10.1111/j.1460-9568.2008.06489.x

[81] A. S. Farb, Z. V. Segal, H. Mayberg, J. Bean, D. McKeon, Z. Fatima and A. K. Anderson, "Attending to the Present:
Mindfulness Mediation Reveals Distinct Neural Modes of Self-Reference," Social Cognitive and Affective Neuroscience, Vol. 2, No. 4, 2007, pp. 313-322. doi: $10.1093 / \mathrm{scan} / \mathrm{nsm} 030$

[82] J. D. Teasdale, "Emotional Processing, Three Modes of Mind and the Prevention of Relapse in Depression," Behaviour Research and Therapy, Vol. 37, No. 1, 1999, pp. 53-77. doi:10.1016/S0005-7967(99)00050-9

[83] D. J. Siegel, "Emotion as Integration: A Possible Answer to the Question What Is Emotion," In: D. Fosha, D. J. Siegel and M. F. Solomon, Eds., The Healing Power of Emotion: Affective Neuroscience, Development and Clinical Practice, Norton, New York, 2009, pp. 145-171.

[84] M. B. Stein, A. N. Simmons, J. S. Feinstein and M. P. Paulus, "Increased Amygdala and Insula Activation during Emotion Processing in Anxiety-Prone Subjects," American Journal of Psychiatry, Vol. 164, No. 2, 2007, pp. 318-327. doi:10.1176/appi.ajp.164.2.318

[85] N. B. Schmidt, M. J. Zvolensky and J. K. Maner, “Anxiety Sensitivity: Prospective Prediction of Panic Attacks and AXIS I Pathology," Journal of Psychiatric Research, Vol. 40, No.8, 2006, pp. 691-699. doi:10.1016/j.jpsychires.2006.07.009

[86] L. Greenberg, "Emotion-Focused Therapy: Coaching Clients to Work through Their Feelings," American Psychological Association, Washington DC, 2002. doi:10.1037/10447-000

[87] S. C. Hayes, K. D. Strosahl and K. G. Wilson, “Acceptance and Commitment Therapy: An Experiential Approach to Behavior Change," Guilford Press, New York, 1999.

[88] J. W. Griffith, R. E. Zinbarg, M. G. Craske, S. Mineka, R. D. Rose, A. M. Waters and J. M. Sutton, "Neuroticism as a Common Dimension in the Internalizing Disorders," Psychological Medicine, Vol. 40, No. 7, 2010, pp. 11251136. doi:10.1017/S0033291709991449

[89] T. A. Brown and D. H. Barlow, "A Proposal for a Dimensional Classification System Based on the Shared Features of the DSM-IV Anxiety and Mood Disorders: Implications for Assessment and Treatment," Psychological Assessment, Vol. 21, No. 3, 2009, pp. 256-271. doi: $10.1037 / \mathrm{a} 0016608$

[90] M. P. Paulus, C. Rogalsky and A. Simmons, "Increased Activation in the Right Insula during Risk-Taking Decision Making Is Related to Harm Avoidance and Neuroticism," NeuroImage, Vol. 19, No. 4, 2003, pp. 1439-1448. doi:10.1016/S1053-8119(03)00251-9

[91] T. Deckersbach, D. D. Dougherty and S. L. Rauch, "Functional Imaging of Mood and Anxiety Disorders," Journal of Neuroimaging, Vol. 16, No. 1, 2006, pp. 1-10. doi: $10.1177 / 1051228405001474$

[92] H. R. Cremers, L. R. Demenescu, A. Aleman, R. Renken, M. J. Van Tol, N. J. Van der Wee, D. J. Veltman and K. Roelofs, "Neuroticism Modulates Amygdala-Prefrontal Connectivity in Response to Negative Emotional Facial Expressions," NeuroImage, Vol. 49, No. 1, 2010, pp. 963-970. doi:10.1016/j.neuroimage.2009.08.0233

[93] S. H. Kim, J. H. Hwang, H. S. Park and S. E. Kim, "Rest- 
ing Brain Metabolic Correlates of Neuroticism and Extraversion in Young Men," Neuroreport, Vol. 19, No. 8, 2008, pp. 883-886. doi:10.1097/WNR.0b013e328300080f

[94] S. Grimm, J. Ernst, P. Boesiger, D. Schuepbach, D. Hell, H. Boeker and G. Northoff, "Increased Self-Focus in Major Depressive Disorder Is Related to Neural Abnormalities in Subcortical-Cortical Midline Structures," $\mathrm{Hu}$ man Brain Mapping, Vol. 30, No. 8, 2009, pp. 2617-2627. doi:10.1002/hbm.20693

[95] J. Wacker, D. G. Dillon and D. A. Pizzagalli, "The Role of the Nucleus Accumbens and Rostral Anterior Cingulate Cortex in Anhedonia: Integration of Resting EEG, fMRI, and Volumetric Techniques," NeuroImage, Vol. 46, No. 1, 2009, pp. 327-337.

doi:10.1016/j.neuroimage.2009.01.058

[96] H. S. Mayberg, S. K. Brannan, R. K. Mahurin, P. A. Jerabek, J. S. Brickman, J. L. Tekell, J. A. Silva, S. McGinnis, T. G. Glass, C. C. Martin and P. T. Fox, "Cingulate Function in Depression: A Potential Predictor of Treatment Response," Neuroreport, Vol. 8, No. 4, 1997, pp. 1057-
1061. doi:10.1097/00001756-199703030-00048

[97] A. S. Korb, A. M. Hunter, I. A. Cook and A. F. Leuchter, "Rostral Anterior Cingulate Cortex Theta Current Density and Response to Antidepressants and Placebo in Major Depression," Clinical Neurophysiology, Vol. 120, No. 7, 2009, pp. 1313-1319. doi:10.1016/j.clinph.2009.05.008

[98] T. D. Wager, V. A. Van Ast, B. L. Hughes, M. L. Davidson, M. A. Lindquist and K. N. Ochsner, "Brain Mediators of Cardiovascular Responses to Social Threat, Part II: Prefrontal-Subcortical Pathways and Relationship with Anxiety," NeuroImage, Vol. 47, No. 3, 2009, pp. 836-851. doi:10.1016/j.neuroimage.2009.05.044

[99] J. Mohlman, R. B. Price, D. A. Eldreth, D. Chazina, D. M. Glover and W. R. Kate, “ The Relation of Worry to Prefrontal Cortex Volume in Older Adults with and without Generalized Anxiety Disorder," Psychiatry Research: Neuroimaging, Vol. 173, No. 2, 2009, pp. 121-127. doi:10.1016/j.pscychresns.2008.09.010 